\title{
Microwave assisted regioselective cycloaddition reactions between 9-substituted anthracenes and levoglucosenone
}

Ariel M. Sarotti, ${ }^{\dagger}$ Madeleine M. Joullié, ${ }^{* *}{ }^{\star \star}$ Rolando A. Spanevello ${ }^{\dagger}$ and Alejandra G. Suárez ${ }^{* \dagger}$

†'Instituto de Química Orgánica de Síntesis, Facultad de Ciencias Bioquímicas y Farmacéuticas, Universidad Nacional de Rosario - CONICET Suipacha 531, S2002LRK Rosario, Argentina

Department of Chemistry, University of Pennsylvania, 231 S. 34th Street, Philadelphia, PA 19104 USA.

asuarez@fbioyf.unr.edu.ar

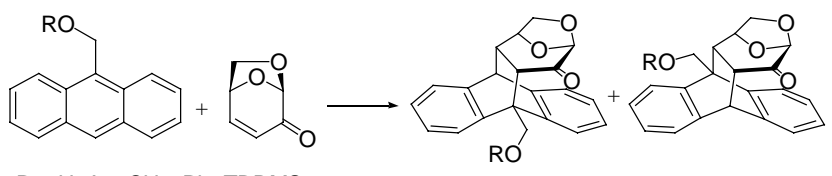

$\mathrm{R}=\mathrm{H}, \mathrm{Ac}, \mathrm{CH}_{3}, \mathrm{Ph}, \mathrm{TBDMS}$ 


\section{General}

The microwave heating was performed in a CEM-Discover ${ }^{\circledR}$ System using a $10 \mathrm{~mL}$ pressure-rated reaction vials. The melting points were taken on a Leitz Wetzlar Microscope Heating Stage Model 350 apparatus and are uncorrected. Optical rotations were recorded with a Jasco DIP 1000 polarimeter. Infrared spectra were obtained on an IRPrestige-21 Fourier Transform Spectrophotometer Shimadzu. High resolution mass spectra (HRMS) were obtained on a Micromass AutoSpec. Nuclear magnetic resonance spectra were recorded on a Bruker AC-200, a Bruker Avance-300 or a Bruker AMX500 spectrometers with tetramethylsilane as internal standard and deuterochloroform as solvent. The NMR assignments were corroborated by NOE measurements, $\mathrm{H}, \mathrm{H}-$ and $\mathrm{H}, \mathrm{C}$-correlations.

The reactions were monitored by thin layer chromatography carried out on $0.25 \mathrm{~mm}$ E. Merck silica gel plates (60F254) that were developed using UV light and anisaldehydesulfuric acid-acetic acid with subsequent heating. Flash column chromatography were performed using Merck silica gel $60 \mathrm{H}$, by gradient elution created by mixtures of hexanes and increasing amounts of ethyl acetate.

\section{Levoglucosenone (1)}

Levoglucosenone was synthesized according to the procedure described in literature. ${ }^{1}$

\section{9-(Methoxymethyl)anthracene (3a)}

This compound was prepared by a modification of the published procedure. ${ }^{2}$ Anthracen9-ylmethanol $97 \%$ (6.09 g, $28.38 \mathrm{mmol})$ was dried azeotropically with dry benzene and dissolved in THF $(96 \mathrm{~mL})$ under argon. This solution was cooled at $0{ }^{\circ} \mathrm{C}$ and $\mathrm{NaH}$ $(60 \%, 3.51 \mathrm{~g}, 87.83 \mathrm{mmol})$ was incorporated and stirred for 20 minutes. Methyl iodide $(4.4 \mathrm{~mL}, 70.71 \mathrm{mmol})$ and tetrabutyl ammonium iodide $(1.04 \mathrm{~g}, 2.82 \mathrm{mmol})$ were added, the final solution was stirred for 40 minutes and allowed to reach room temperature. This solution was cooled at $0{ }^{\circ} \mathrm{C}$ and carefully added methanol $(5 \mathrm{~mL})$ and diluted with AcOEt $(1 \mathrm{~L})$. The organic phase was washed with water $(2 \times 100 \mathrm{~mL})$, brine $(100 \mathrm{~mL})$, dried $\left(\mathrm{Na}_{2} \mathrm{SO}_{4}\right)$ and concentrated. The residual solid was purified by flash chromatography to afford 3a $(6.06 \mathrm{~g}, 27.25 \mathrm{mmol}, 96 \%)$.

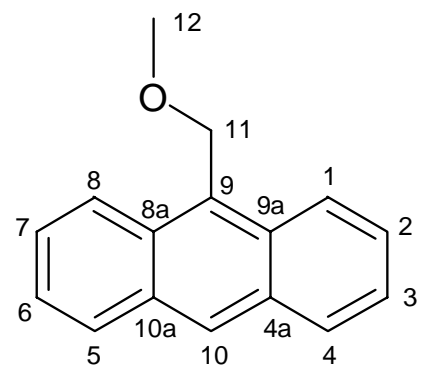

3a

Yellow crystalline solid; mp 91-92 ${ }^{\circ} \mathrm{C}$ (hexane/ethyl acetate); IR (KBr) 2977, 2924, $1623,1447,1344,1188,1087,940,894,738,729 \mathrm{~cm}^{-1} ;{ }^{1} \mathrm{H}$ NMR $\left(\mathrm{CDCl}_{3}\right) \delta 8.42(\mathrm{~s}, 1$ H, H-10), 8.36 (d, J=8.8 Hz, $2 \mathrm{H}, \mathrm{H}-1$ and H-8), 7.98 (d, J=8.4 Hz, $2 \mathrm{H}, \mathrm{H}-4$ and H-5), 7.56-7.40 (m, 4 H, H-2 H-3, H-6 and H-7), 5.40 (s, 2 H, H-11), 3.51 (s, $3 \mathrm{H}, \mathrm{H}-12) ;{ }^{13} \mathrm{C}$

\footnotetext{
${ }^{1}$ Witczak, Z. J., Ed. Levoglucosenone and Levoglucosans: Chemistry and Applications; ATL Press: Mount Prospect, 1994, Chapter 2 (Morin, C.).

2 Zimmermman, C.; Mohr, M.; Zipse, H.; Eichberger, R.; Schnabel, W. J. Photochem. Photobiol. A. 1999, 125, 47.
} 
NMR $\left(\mathrm{CDCl}_{3}\right) \delta 131.3(\mathrm{C}, \mathrm{C}-9), 130.8(\mathrm{C}, \mathrm{C}-4 \mathrm{a}$ and C-10a), $128.8(\mathrm{CH}, \mathrm{C}-4$ and C-5), 128.5 (C, C-8a and C-9a), 128.2 (CH, C-10), 126.0 (CH, C-1 and C-8), 124.8 (CH, C-2 and C-7), $124.1(\mathrm{CH}, \mathrm{C}-3$ and $\mathrm{C}-6), 66.4\left(\mathrm{CH}_{2}, \mathrm{C}-11\right), 58.1\left(\mathrm{CH}_{3}, \mathrm{C}-12\right)$.

\section{(Anthracen-9-ylmethoxy)(tert-butyl)dimethylsilane (3b)}

Anthracen-9-ylmethanol 97\% (4.45 g, $20.73 \mathrm{mmol})$ was dissolved in dry THF (125 mL) under argon and dry triethylamine $(8.56 \mathrm{~mL}, 61.53 \mathrm{mmol})$, DMAP $(0.26 \mathrm{~g}, 2.08 \mathrm{mmol})$ and TBDMSCl $97 \%(8.13 \mathrm{~g}, 52.32 \mathrm{mmol})$ were added in this order. The solution was stirred for 40 hours at room temperature and diluted with AcOEt $(1 \mathrm{~L})$. The organic phase was washed with water $(1 \times 280 \mathrm{~mL}), \mathrm{NaHCO}_{3} 5 \%(1 \times 280 \mathrm{~mL}), \mathrm{NH}_{4} \mathrm{Cl}$ sat $(1 \mathrm{x}$ $280 \mathrm{~mL})$ and brine $(1 \times 280 \mathrm{~mL})$, dried $\left(\mathrm{Na}_{2} \mathrm{SO}_{4}\right)$ and concentrated. The residual solid was purified by flash chromatography to afford $\mathbf{3 b}$ (6.53 g, $20.25 \mathrm{mmol}, 98 \%)$.

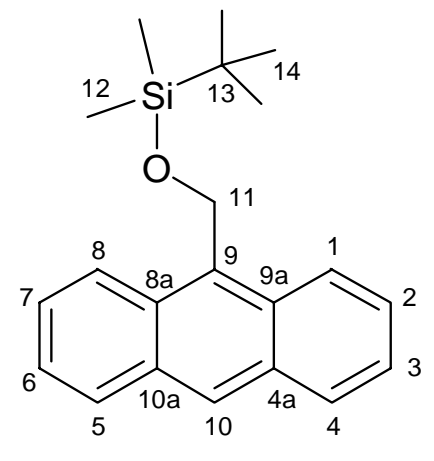

$3 b$

Pale green crystalline solid; mp $115-116^{\circ} \mathrm{C}$ (hexane); IR (KBr) 3062, 2956, 2950, 2853 , $1461,1345,1251,1072,1036,833,779 \mathrm{~cm}^{-1} ;{ }^{1} \mathrm{H}$ NMR $\left(\mathrm{CDCl}_{3}\right) \delta 8.40$ (s, $\left.1 \mathrm{H}, \mathrm{H}-10\right)$, 8.38 (d, J=8.0 Hz, $2 \mathrm{H}, \mathrm{H}-1$ and H-8), 7.98 (d, J=8.0 Hz, $2 \mathrm{H}, \mathrm{H}-4$ and H-5), 7.56-7.40 (m, 4 H, H-2, H-3, H-6 and H-7), 5.64 (s, 2 H, H-11), 0.91 (s, 9 H, H-14), 0.10 (s, 6 H, $\mathrm{H}-12) ;{ }^{13} \mathrm{C}$ NMR $\left(\mathrm{CDCl}_{3}\right) \delta 131.4(\mathrm{C}, \mathrm{C}-9), 130.2$ (C, C-8a and C-9a), 128.8 (CH, C-4 and $\mathrm{C}-5), 128.8(\mathrm{C}, \mathrm{C}-4 \mathrm{a}$ and $\mathrm{C}-10 \mathrm{a}), 127.7$ (CH, C-10), 125.7 (CH, C-1 and C-8), $124.7(\mathrm{CH}, \mathrm{C}-2$ and $\mathrm{C}-7), 124.4(\mathrm{CH}, \mathrm{C}-3$ and $\mathrm{C}-6), 57.8\left(\mathrm{CH}_{2}, \mathrm{C}-11\right), 25.9\left(\mathrm{CH}_{3}, \mathrm{C}-\right.$ $14), 18.3(\mathrm{C}, \mathrm{C}-13),-5.1\left(\mathrm{CH}_{3}, \mathrm{C}-12\right)$.

\section{9-(Phenoxymethyl)anthracene (3c)}

Anthracen-9-ylmethanol 97\% (2.81 g, $13.07 \mathrm{mmol})$ was dried azeotropically with dry benzene and dissolved in dry $\mathrm{CH}_{2} \mathrm{Cl}_{2}(200 \mathrm{~mL})$ under argon. This solution was cooled at $0{ }^{\circ} \mathrm{C}$ and $\mathrm{NEt}_{3}(2.20 \mathrm{~mL}, 15.79 \mathrm{mmol})$ and $\mathrm{MsCl}(1.23 \mathrm{~mL}, 15.89 \mathrm{mmol})$ were added in this order and the reaction stirred for 5 minutes. The solution was diluted with $\mathrm{CH}_{2} \mathrm{Cl}_{2}$ $(200 \mathrm{~mL})$ and washed with water $(100 \mathrm{~mL})$, brine $(100 \mathrm{~mL})$, dried $\left(\mathrm{Na}_{2} \mathrm{SO}_{4}\right)$ and concentrated. The residual solid was dried azeotropically with dry benzene and dissolved in dry acetone $(360 \mathrm{~mL})$. Phenol (4.68 g, $49.8 \mathrm{mmol})$ and anhydrous $\mathrm{K}_{2} \mathrm{CO}_{3}$ $(6.90 \mathrm{~g}, 49.9 \mathrm{mmol})$ were incorporated and the solution was stirred at reflux for 24 hours under argon. The solvent was evaporated under reduced pressure. Water $(300 \mathrm{~mL})$ and $\mathrm{CH}_{2} \mathrm{Cl}_{2}(300 \mathrm{~mL})$ were added. The aqueous phase was extracted with $\mathrm{CH}_{2} \mathrm{Cl}_{2}(3 \mathrm{x}$ $100 \mathrm{~mL})$. The organic phase was washed with saturated $\mathrm{Na}_{2} \mathrm{CO}_{3}(2 \times 150 \mathrm{~mL})$, brine $(2$ x $150 \mathrm{~mL})$, dried $\left(\mathrm{Na}_{2} \mathrm{SO}_{4}\right)$ and concentrated. The residual solid was purified by flash chromatography to afford 3c (3.38 g, $11.89 \mathrm{mmol}, 91 \%)$. 


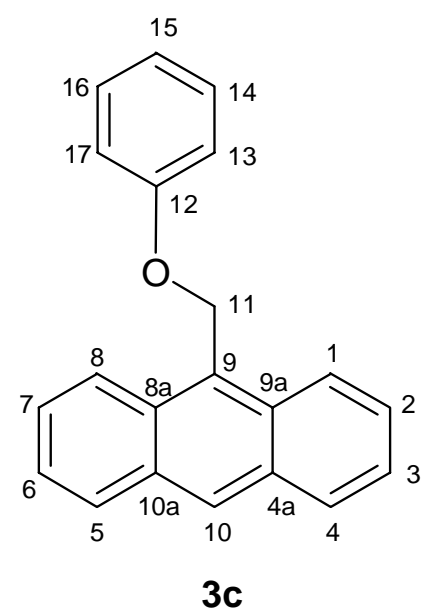

Orange solid; mp 117-118 ${ }^{\circ} \mathrm{C}$ (EtOH); IR (KBr) 3031, 2886, 1597, 1585, 1494, 1476, 1236, 989, 879, $731 \mathrm{~cm}^{-1}$; ${ }^{1} \mathrm{H}$ NMR $\left(\mathrm{CDCl}_{3}\right) \delta 8.49(\mathrm{~s}, 1 \mathrm{H}, \mathrm{H}-10), 8.29-8.24(\mathrm{~m}, 2 \mathrm{H}$, H-1 and H-8), 8.04-7.99 (m, 2 H, H-4 and H-5), 7.55-7.00 (m, 9 H, H-2, H-3, H-6, H-7, H-13, H-14, H-15, H-16 and H-17), 5.92 (s, $2 \mathrm{H}, \mathrm{H}-11) ;{ }^{13} \mathrm{C} \mathrm{NMR}\left(\mathrm{CDCl}_{3}\right) \delta 159.1(\mathrm{C}$, C-12), 131.4 (C, C-9), 130.9 (C, C-4a and C-10a), 129.5 (CH, C-4 and C-5), 129.0 (CH, C-1 and C-8), 128.9 (C, C-8a and C-9a), 126.8 (CH, C-14 and C-16), $126.4(\mathrm{CH}, \mathrm{C}-2$ and C-7), 124.9 (CH, C-3 and C-6), 123.9 (CH, C-10), $121.1(\mathrm{CH}, \mathrm{C}-15), 114.7(\mathrm{CH}, \mathrm{C}-$ 13 and $\mathrm{C}-17), 62.5\left(\mathrm{CH}_{2}, \mathrm{C}-11\right)$.

\section{Anthracen-9-ylmethylacetate (3d)}

Anthracen-9-ylmethanol 97\% (1.08 g, $5.03 \mathrm{mmol})$ was dissolved in dry $\mathrm{CH}_{2} \mathrm{Cl}_{2}(44 \mathrm{~mL})$ under argon. The solution was cooled at $0{ }^{\circ} \mathrm{C}$ and dry pyridine $(2.04 \mathrm{~mL}, 25.22 \mathrm{mmol})$, DMAP (63 mg, $0.50 \mathrm{mmol})$ and $\mathrm{Ac}_{2} \mathrm{O}(1.46 \mathrm{~mL}, 15.47 \mathrm{mmol})$ were added in this order. The solution was stirred for 1 hour at $0{ }^{\circ} \mathrm{C}$. The solvent was evaporated under reduced pressure and the crude was dissolved with AcOEt $(400 \mathrm{~mL})$. The organic phase was washed with saturated $\mathrm{CuSO}_{4}(3 \times 100 \mathrm{~mL})$ and brine $(2 \times 100 \mathrm{~mL})$, dried $\left(\mathrm{Na}_{2} \mathrm{SO}_{4}\right)$ and concentrated. The residual solid was purified by flash chromatography to afford $\mathbf{3 d}$ (1.15 g, $4.59 \mathrm{mmol}, 91 \%)$.

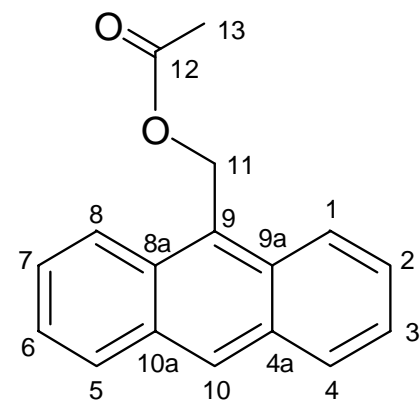

$3 d$

Pale yellow crystalline solid; mp $115-116^{\circ} \mathrm{C}$ (hexane); IR (KBr) 3058, 3001, 2921, $1725(\mathrm{C}=\mathrm{O}), 1447,1380,1250,1027,890,738 \mathrm{~cm}^{-1} ;{ }^{1} \mathrm{H}$ NMR $\left(\mathrm{CDCl}_{3}\right) \delta 8.48(\mathrm{~s}, 1 \mathrm{H}$, H-10), 8.32 (d, $J=8,8 \mathrm{~Hz}, 2 \mathrm{H}, \mathrm{H}-1$ and H-8), 8.01 (d, $J=8,4 \mathrm{~Hz}, 2 \mathrm{H}, \mathrm{H}-4$ and H-5), 7.59-7.45 (m, 4 H, H-2, H-3, H-6 and H-7), 6.13 (s, $2 \mathrm{H}, \mathrm{H}-11), 2.07$ (s, $3 \mathrm{H}, \mathrm{H}-13$ ); ${ }^{13} \mathrm{C}$ NMR $\left(\mathrm{CDCl}_{3}\right) \delta 171.2(\mathrm{C}, \mathrm{C}-12), 131.3$ (C, C-9), 130.9 (C, C-4a and C-10a), 129.1 (CH, C-10), $129.0(\mathrm{CH}, \mathrm{C}-4$ and C-5), 126.5 (CH, C-3 and C-6), 126.1 (C, C-8a and C- 
9a), $125.0\left(\mathrm{CH}, \mathrm{C}-2\right.$ and C-7), $123.8(\mathrm{CH}, \mathrm{C}-1$ and $\mathrm{C}-8), 58.7\left(\mathrm{CH}_{2}, \mathrm{C}-11\right), 20.9\left(\mathrm{CH}_{3}\right.$, C-13).

General procedure for the cycloaddition reaction of levoglucosenone and 9substituted anthracenes. Thermal conditions.

Levoglucosenone and the corresponding diene (2.7 equiv) were dissolved in toluene ( $c$ $0.23 \mathrm{M}$ ) and stirred at reflux for the time indicated in Table 1. The solvent was evaporated under reduced pressure and the residue was purified by flash chromatography.

General procedure for the cycloaddition reaction of levoglucosenone and 9substituted anthracenes. Microwave conditions.

Levoglucosenone $(80 \mathrm{mg}, 0.63 \mathrm{mmol})$ and the corresponding diene in the amount indicated in Tables 2-4 were placed in a $10 \mathrm{~mL}$ vial and dissolved in the corresponding solvent $(0.46 \mathrm{~mL})$ and heated for the time and power indicated in Tables 2-4. The solvent was evaporated under reduced pressure and the residue was purified by flash chromatography.

Diels-Alder Product 4a

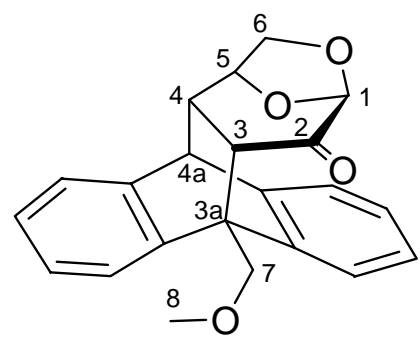

$4 a$

White crystalline solid; mp 251-252 ${ }^{\circ} \mathrm{C}$ (hexane/chloroform); $[\alpha]^{29}{ }_{\mathrm{D}}-43.8$ (c 0.88 , $\left.\mathrm{CHCl}_{3}\right)$; IR (KBr) 3069, 2927, $1719(\mathrm{C}=\mathrm{O}), 1464,1457,1115,993,921,770 \mathrm{~cm}^{-1} ;{ }^{1} \mathrm{H}$ NMR $\left(\mathrm{CDCl}_{3}\right) \delta$ 7.51-7.00 (m, $8 \mathrm{H}$, aromatics), $4.89\left(\mathrm{~d}, J_{\text {gem }}=9.5 \mathrm{~Hz}, 1 \mathrm{H}, \mathrm{H}-7\right), 4.77(\mathrm{~d}$, $\left.J_{5-6 \mathrm{exo}}=4.9 \mathrm{~Hz}, 1 \mathrm{H}, \mathrm{H}-5\right), 4.62\left(\mathrm{~d}, J_{\mathrm{gem}}=9.5 \mathrm{~Hz}, 1 \mathrm{H}, \mathrm{H}-7\right), 4.54(\mathrm{~s}, 1 \mathrm{H}, \mathrm{H}-1), 4.27(\mathrm{~s}, 1$ $\mathrm{H}, \mathrm{H}-4 \mathrm{a}), 3.76-3.61$ (m, $5 \mathrm{H}, \mathrm{H}-6$ endo, H-6 exo and H-8), 3.10 (d, $J_{3-4}=9.7 \mathrm{~Hz}, 1 \mathrm{H}, \mathrm{H}-$ 3), $2.24\left(\mathrm{~d}, J_{3-4}=9.7 \mathrm{~Hz}, 1 \mathrm{H}, \mathrm{H}-4\right) ;{ }^{13} \mathrm{C} \mathrm{NMR}\left(\mathrm{CDCl}_{3}\right) \delta 199.1(\mathrm{C}, \mathrm{C}-2), 145.2(\mathrm{C}$, aromatic), 141.3 (C, aromatic), 140.9 (C, aromatic), 140.6 (C, aromatic), $126.2(\mathrm{CH}$, aromatic), $126.0(\mathrm{CH}, 2 \mathrm{C}$, aromatic), $125.8(\mathrm{CH}$, aromatic), $125.0(\mathrm{CH}$, aromatic), $124.2(\mathrm{CH}$, aromatic), $122.0(\mathrm{CH}$, aromatic), $121.3(\mathrm{CH}$, aromatic $), 99.8(\mathrm{CH}, \mathrm{C}-1)$, $77.1(\mathrm{CH}, \mathrm{C}-5), 70.5\left(\mathrm{CH}_{2}, \mathrm{C}-7\right), 68.7\left(\mathrm{CH}_{2}, \mathrm{C}-6\right), 59.0\left(\mathrm{CH}_{3}, \mathrm{C}-8\right), 50.3(\mathrm{CH}, \mathrm{C}-4 \mathrm{a})$, $49.8(\mathrm{C}, \mathrm{C}-3 \mathrm{a}), 44.7(\mathrm{CH}, \mathrm{C}-4), 44.0(\mathrm{CH}, \mathrm{C}-3)$; HRMS calc. for $\mathrm{C}_{22} \mathrm{H}_{20} \mathrm{O}_{4} \mathrm{Na}[\mathrm{M}+\mathrm{Na}]^{+}$ 371.1259. Found 371.1266.

\section{Diels-Alder Product 5a}

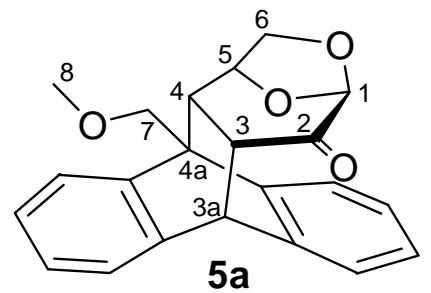


White crystalline solid; mp 236-237 ${ }^{\circ} \mathrm{C}$ (hexane/chloroform); $[\alpha]^{14}{ }_{\mathrm{D}}-127.7$ (c 0.92 , $\left.\mathrm{CHCl}_{3}\right)$; IR (KBr) 3062, 2969, $1735(\mathrm{C}=\mathrm{O}), 1457,1118,1108,983,746 \mathrm{~cm}^{-1} ;{ }^{1} \mathrm{H}$ NMR $\left(\mathrm{CDCl}_{3}\right) \delta 7.36-7.03(\mathrm{~m}, 8 \mathrm{H}$, aromatics $), 4.88\left(\mathrm{~d}, J_{5-6 \mathrm{ex} 0}=5.4 \mathrm{~Hz}, 1 \mathrm{H}, \mathrm{H}-5\right), 4.84\left(\mathrm{~d}, J_{3-}\right.$ $3 \mathrm{a}=3.4 \mathrm{~Hz}, 1 \mathrm{H}, \mathrm{H}-3 \mathrm{a}), 4.69$ (d, $\left.J_{\text {gem }}=9.9 \mathrm{~Hz}, 1 \mathrm{H}, \mathrm{H}-7\right), 4.64(\mathrm{~s}, 1 \mathrm{H}, \mathrm{H}-1), 4.62$ (d, $\left.J_{\text {gem }}=9.9 \mathrm{~Hz}, 1 \mathrm{H}, \mathrm{H}-7\right), 3.84\left(\mathrm{dd}, J_{\text {gem }}=7.2 \mathrm{~Hz}, J_{5-6 \text { exo }}=5.4 \mathrm{~Hz}, 1 \mathrm{H}, \mathrm{H}-6\right.$ exo $), 3.72-3.68$ $\left(\mathrm{m}, 4 \mathrm{H}, \mathrm{H}-6\right.$ endo and H-8), $2.99\left(\mathrm{dd}, J_{3-4}=9.9 \mathrm{~Hz}, J_{3-3 \mathrm{a}}=3.4 \mathrm{~Hz}, 1 \mathrm{H}, \mathrm{H}-3\right), 2.42\left(\mathrm{~d}, J_{3-}\right.$ $\left.{ }_{4}=9.9 \mathrm{~Hz}, 1 \mathrm{H}, \mathrm{H}-4\right) ;{ }^{13} \mathrm{C} \mathrm{NMR}\left(\mathrm{CDCl}_{3}\right) \delta 199.0(\mathrm{C}, \mathrm{C}-2), 144.5$ (C, aromatic), $141.5(\mathrm{C}$, aromatic), 141.2 (C, aromatic), $140.3(\mathrm{C}$, aromatic), $126.2(\mathrm{CH}$, aromatic), $125.9(\mathrm{CH}$, $3 \mathrm{C}$, aromatic), $124.9(\mathrm{CH}$, aromatic $), 124.0(\mathrm{CH}$, aromatic $), 121.8(\mathrm{CH}$, aromatic $), 121.4$ (CH, aromatic), $99.4(\mathrm{CH}, \mathrm{C}-1), 72.7(\mathrm{CH}, \mathrm{C}-5), 70.4\left(\mathrm{CH}_{2}, \mathrm{C}-7\right), 69.4\left(\mathrm{CH}_{2}, \mathrm{C}-6\right)$, $59.5\left(\mathrm{CH}_{3}, \mathrm{C}-8\right), 47.7(\mathrm{C}, \mathrm{C}-4 \mathrm{a}), 46.3(\mathrm{CH}, \mathrm{C}-3 \mathrm{a}), 45.4(\mathrm{CH}, \mathrm{C}-4), 43.7(\mathrm{CH}, \mathrm{C}-3)$; HRMS calc. for $\mathrm{C}_{22} \mathrm{H}_{20} \mathrm{O}_{4}[\mathrm{M}]^{+}$348.1362. Found 348.1374.

\section{Diels-Alder Product 4b}

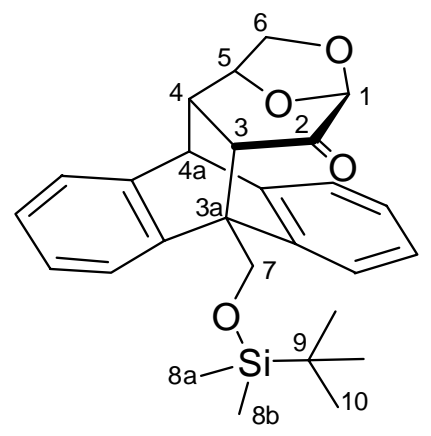

4b

Pale yellow oil; $[\alpha]^{21}$ - 31.1 ( c 1.41, $\mathrm{CHCl}_{3}$ ); IR (film) 3072, 3023, 2955, 2856, 1724 $(\mathrm{C}=\mathrm{O}), 1457,1255,1107,833,765 \mathrm{~cm}^{-1} ;{ }^{1} \mathrm{H}$ NMR $\left(\mathrm{CDCl}_{3}\right) \delta 7.65-6.98(\mathrm{~m}, 8 \mathrm{H}$, aromatics), 5.28 (d, $\left.J_{\text {gem }}=10.1 \mathrm{~Hz}, 1 \mathrm{H}, \mathrm{H}-7\right), 4.85-4.80(\mathrm{~m}, 2 \mathrm{H}, \mathrm{H}-7$ y H-5), 4.55 (s, 1 H, H-1), 4.28 (d, $\left.J_{4-4 \mathrm{a}}=1.5 \mathrm{~Hz}, 1 \mathrm{H}, \mathrm{H}-4 \mathrm{a}\right), 3.78\left(\mathrm{dd}, J_{\text {gem }}=7.2 \mathrm{~Hz}, J_{5-6 \mathrm{exo}}=5.2 \mathrm{~Hz}, 1 \mathrm{H}, \mathrm{H}-\right.$ 6 exo), $3.72\left(\mathrm{dd}, J_{\mathrm{gem}}=7.2 \mathrm{~Hz}, J_{5 \text {-6endo }}=1.3 \mathrm{~Hz}, 1 \mathrm{H}, \mathrm{H}-6\right.$ endo $), 3.16\left(\mathrm{~d}, J_{3-4}=9.7 \mathrm{~Hz}, 1 \mathrm{H}\right.$, H-3), 2.32 (dd, $\left.J_{3-4}=9.7 \mathrm{~Hz}, J_{4-4 \mathrm{a}}=1.5 \mathrm{~Hz}, 1 \mathrm{H}, \mathrm{H}-4\right), 0.96$ (s, $\left.9 \mathrm{H}, \mathrm{H}-10\right), 0.32$ (s, $3 \mathrm{H}, \mathrm{H}-$ 8a), 0.24 (s, $3 \mathrm{H}, \mathrm{H}-8 \mathrm{~b}) ;{ }^{13} \mathrm{C} \mathrm{NMR}\left(\mathrm{CDCl}_{3}\right) \delta 198.8$ (C, C-2), 145.6 (C, aromatic), 141.6 $(\mathrm{C}$, aromatic), $141.1(\mathrm{C}$, aromatic), $140.8(\mathrm{C}$, aromatic), $126.0(\mathrm{CH}$, aromatic), 125.7 $(\mathrm{CH}$, aromatic), $125.6(\mathrm{CH}, 2 \mathrm{C}$, aromatic), $124.9(\mathrm{CH}$, aromatic $), 124.7(\mathrm{CH}$, aromatic), $121.9(\mathrm{CH}$, aromatic), $121.3(\mathrm{CH}$, aromatic), $99.9(\mathrm{CH}, \mathrm{C}-1), 77.3(\mathrm{CH}, \mathrm{C}-5), 68.7$ $\left(\mathrm{CH}_{2}, \mathrm{C}-6\right), 61.2\left(\mathrm{CH}_{2}, \mathrm{C}-7\right), 50.8$ (C, C-3a), $50.4(\mathrm{CH}, \mathrm{C}-4 \mathrm{a}), 44.8(\mathrm{CH}, \mathrm{C}-4), 43.0$ (CH, C-3), $25.8\left(\mathrm{CH}_{3}, \mathrm{C}-10\right), 18.3(\mathrm{C}, \mathrm{C}-9),-5.4\left(\mathrm{CH}_{3}, \mathrm{C}-8 \mathrm{a}\right),-5.6\left(\mathrm{CH}_{3}, \mathrm{C}-8 \mathrm{~b}\right) ; \mathrm{HRMS}$ calc. for $\mathrm{C}_{27} \mathrm{H}_{32} \mathrm{O}_{4} \mathrm{SiNa}[\mathrm{M}+\mathrm{Na}]^{+}$471.1968. Found 471.1827 .

\section{Diels-Alder Product 5b}

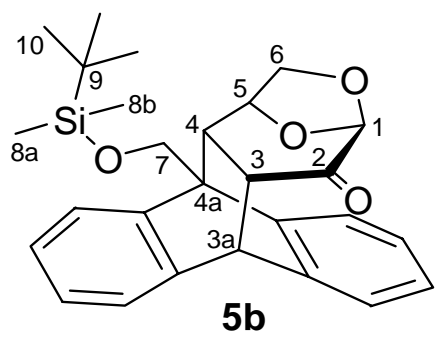


Pale yellow solid; mp $162-163{ }^{\circ} \mathrm{C}$ (hexane); $[\alpha]^{28}{ }_{\mathrm{D}}-76.8\left(c 0.64, \mathrm{CHCl}_{3}\right)$; IR $(\mathrm{KBr})$ 3005, 2951, $1726(\mathrm{C}=\mathrm{O}), 1471,1257,1142,1108,990,835,772 \mathrm{~cm}^{-1} ;{ }^{1} \mathrm{H}$ NMR $\left(\mathrm{CDCl}_{3}\right) \delta$ 7.45-7.32 (m, $2 \mathrm{H}$, aromatics), 7.20-7.03 (m, $6 \mathrm{H}$, aromatics), 4.94 (s, $2 \mathrm{H}, \mathrm{H}-$ 7), $4.88\left(\mathrm{~d}, J_{5-6 e x 0}=5.2 \mathrm{~Hz}, 1 \mathrm{H}, \mathrm{H}-5\right), 4.84\left(\mathrm{~d}, J_{3-3 \mathrm{a}}=3.4 \mathrm{~Hz}, 1 \mathrm{H}, \mathrm{H}-3 \mathrm{a}\right), 4.65(\mathrm{~s}, 1 \mathrm{H}, \mathrm{H}-$ 1), $3.80\left(\mathrm{dd}, J_{\text {gem }}=7.1 \mathrm{~Hz}, J_{5-6 \mathrm{exo}}=5.2 \mathrm{~Hz}, 1 \mathrm{H}, \mathrm{H}-6\right.$ exo $), 3.68\left(\mathrm{dd}, J_{\text {gem }}=7.1 \mathrm{~Hz}, J_{5-}\right.$ 6endo $=1.1 \mathrm{~Hz}, 1 \mathrm{H}, \mathrm{H}-6$ endo), $3.01\left(\mathrm{dd}, J_{3-4}=10.1 \mathrm{~Hz}, J_{3-3 \mathrm{a}}=3.4 \mathrm{~Hz}, 1 \mathrm{H}, \mathrm{H}-3\right), 2.51\left(\mathrm{~d}, J_{3-}\right.$ $\left.{ }_{4}=10.1 \mathrm{~Hz}, 1 \mathrm{H}, \mathrm{H}-4\right), 0.94$ (s, $\left.9 \mathrm{H}, \mathrm{H}-10\right), 0.33$ (s, $\left.3 \mathrm{H}, \mathrm{H}-8 \mathrm{a}\right), 0.25$ (s, $\left.3 \mathrm{H}, \mathrm{H}-8 \mathrm{~b}\right) ;{ }^{13} \mathrm{C}$ NMR $\left(\mathrm{CDCl}_{3}\right) \delta 199.1$ (C, C-2), 144.4 (C, aromatic), 141.7 (C, aromatic), $141.3(\mathrm{C}$, aromatic), $140.5(\mathrm{C}$, aromatic), $126.0(\mathrm{CH}$, aromatic), $125.8(\mathrm{CH}$, aromatic $), 125.7(\mathrm{CH}$, $2 \mathrm{C}$, aromatic), $124.8(\mathrm{CH}$, aromatic), $123.9(\mathrm{CH}$, aromatic $), 122.1(\mathrm{CH}$, aromatic), $121.2\left(\mathrm{CH}\right.$, aromatic), $99.4(\mathrm{CH}, \mathrm{C}-1), 72.8(\mathrm{CH}, \mathrm{C}-5), 69.3\left(\mathrm{CH}_{2}, \mathrm{C}-6\right), 60.1\left(\mathrm{CH}_{2}, \mathrm{C}-\right.$ 7), 48.4 (C, C-4a), $46.3(\mathrm{CH}, \mathrm{C}-3 \mathrm{a}), 45.3(\mathrm{CH}, \mathrm{C}-4), 43.0(\mathrm{CH}, \mathrm{C}-3), 25.6\left(\mathrm{CH}_{3}, \mathrm{C}-10\right)$, 17.9 (C, C-9), -5.6 $\left(\mathrm{CH}_{3}, \mathrm{C}-8 \mathrm{a}\right),-5.8\left(\mathrm{CH}_{3}, \mathrm{C}-8 \mathrm{~b}\right)$; HRMS calc. for $\mathrm{C}_{27} \mathrm{H}_{32} \mathrm{O}_{4} \mathrm{SiNa}$ $[\mathrm{M}+\mathrm{Na}]^{+}$471.1968. Found 471.1960.

\section{Diels-Alder Product 4c}

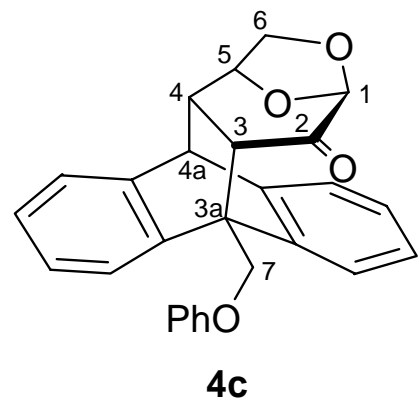

White crystalline solid; mp 249-250 ${ }^{\circ} \mathrm{C}$ (hexane/chloroform); $[\alpha]^{19}{ }_{\mathrm{D}}-64.1$ (c 0.88 , $\mathrm{CHCl}_{3}$ ); IR (KBr) 3068, 3040, 2969, 2899, 1717 (C=O), 1599, 1497, 1464, 1241, 1115, $750 \mathrm{~cm}^{-1} ;{ }^{1} \mathrm{H}$ NMR $\left(\mathrm{CDCl}_{3}\right) \delta 7.47-7.00(\mathrm{~m}, 13 \mathrm{H}$, aromatics $), 5.56\left(\mathrm{~d}, J_{\mathrm{gem}}=9.3 \mathrm{~Hz}, 1\right.$ H, H-7), $5.23\left(\mathrm{~d}, J_{\text {gem }}=9.3 \mathrm{~Hz}, 1 \mathrm{H}, \mathrm{H}-7\right), 4.83\left(\mathrm{~d}, J_{5-6 \mathrm{exo}}=4.3 \mathrm{~Hz}, 1 \mathrm{H}, \mathrm{H}-5\right), 4.57(\mathrm{~s}, 1 \mathrm{H}$, $\mathrm{H}-1), 4.35\left(\mathrm{~d}, J_{4-4 \mathrm{a}}=1.5 \mathrm{~Hz}, 1 \mathrm{H}, \mathrm{H}-4 \mathrm{a}\right), 3.78\left(\mathrm{dd}, J_{\mathrm{gem}}=7.3 \mathrm{~Hz}, J_{5-6 \mathrm{exo}}=4.3 \mathrm{~Hz}, 1 \mathrm{H}, \mathrm{H}-6\right.$ exo $), 3.71\left(\mathrm{dd}, J_{\text {gem }}=7.3 \mathrm{~Hz}, J_{5-6 \text { endo }}=1.0 \mathrm{~Hz}, 1 \mathrm{H}, \mathrm{H}-6\right.$ endo $), 3.25\left(\mathrm{~d}, J_{3-4}=9.7 \mathrm{~Hz}, 1 \mathrm{H}\right.$, $\mathrm{H}-3), 2.37\left(\mathrm{dd}, J_{3-4}=9.7 \mathrm{~Hz}, J_{4-4 \mathrm{a}}=1.5 \mathrm{~Hz}, 1 \mathrm{H}, \mathrm{H}-4\right) ;{ }^{13} \mathrm{C} \mathrm{NMR}\left(\mathrm{CDCl}_{3}\right) \delta 198.8(\mathrm{C}, \mathrm{C}-$ 2), 158.6 (C, aromatic), 145.1 (C, aromatic), 140.8 (C, aromatic), 140.7 (C, aromatic), 140.1 (C, aromatic), $129.5(\mathrm{CH}, 2 \mathrm{C}$, aromatic), $126.4(\mathrm{CH}$, aromatic), $126.1(\mathrm{CH}$, aromatic), $126.0(\mathrm{CH}$, aromatic), $125.9(\mathrm{CH}$, aromatic $), 125.1(\mathrm{CH}$, aromatic), 124.2 $(\mathrm{CH}$, aromatic), $122.2(\mathrm{CH}$, aromatic $), 121.6(\mathrm{CH}$, aromatic $), 121.1(\mathrm{CH}$, aromatic), $114.9\left(\mathrm{CH}, 2 \mathrm{C}\right.$, aromatic), $99.7(\mathrm{CH}, \mathrm{C}-1), 77.1(\mathrm{CH}, \mathrm{C}-5), 68.8\left(\mathrm{CH}_{2}, \mathrm{C}-6\right), 66.2\left(\mathrm{CH}_{2}\right.$, C-7), 50.3 (CH, C-4a), 49.6 (C, C-3a), 44.8 (CH, C-4), 44.3 (CH, C-3); HRMS calc. for $\mathrm{C}_{27} \mathrm{H}_{22} \mathrm{O}_{4}[\mathrm{M}]^{+}$410.1518. Found 410.1500. 


\section{Diels-Alder Product 4d}

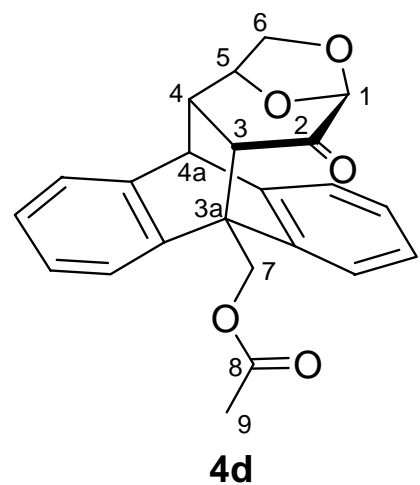

White crystalline solid; mp 241-242 ${ }^{\circ} \mathrm{C}$ (hexane/chloroform); $[\alpha]^{23}{ }_{\mathrm{D}}-51.9$ (c 0.86 , $\mathrm{CHCl}_{3}$ ); IR (KBr) 3069, 3011, 2961, 2909, 1734 (C=O), 1719 (C=O), 1458, 1366, 1236, 1121, 993, 901, 764, $739 \mathrm{~cm}^{-1},{ }^{1} \mathrm{H}$ NMR $\left(\mathrm{CDCl}_{3}\right) \delta$ 7.44-7.07 (m, $8 \mathrm{H}$, aromatics), 5.63 $\left(\mathrm{d}, J_{\text {gem }}=11.5 \mathrm{~Hz}, 1 \mathrm{H}, \mathrm{H}-7\right), 5.33\left(\mathrm{~d}, J_{\text {gem }}=11.5 \mathrm{~Hz}, 1 \mathrm{H}, \mathrm{H}-7\right), 4.82\left(\mathrm{~d}, J_{5-6 \text { exo }}=4.7 \mathrm{~Hz}, 1\right.$ H, H-5), 4.57 (s, $1 \mathrm{H}, \mathrm{H}-1), 4.35$ (d, $\left.J_{4-4 \mathrm{a}}=1.3 \mathrm{~Hz}, 1 \mathrm{H}, \mathrm{H}-4 \mathrm{a}\right), 3.78$ (dd, $J_{\text {gem }}=7.3 \mathrm{~Hz}, J_{5-}$ $6_{\text {exo }}=4.7 \mathrm{~Hz}, 1 \mathrm{H}, \mathrm{H}-6$ exo $), 3.70$ (dd, $J_{\text {gem }}=7.3 \mathrm{~Hz}, J_{5-6 \mathrm{endo}}=1.0 \mathrm{~Hz}, 1 \mathrm{H}, \mathrm{H}-6$ endo), 3.11 $\left(\mathrm{d}, J_{3-4}=9.7 \mathrm{~Hz}, 1 \mathrm{H}, \mathrm{H}-3\right), 2.34\left(\mathrm{dd}, J_{3-4}=9.7 \mathrm{~Hz}, J_{4-4 \mathrm{a}}=1.3 \mathrm{~Hz}, 1 \mathrm{H}, \mathrm{H}-4\right), 2.17(\mathrm{~s}, 3 \mathrm{H}$, $\mathrm{H}-9) ;{ }^{13} \mathrm{C}$ NMR $\left(\mathrm{CDCl}_{3}\right) \delta 198.5$ (C, C-2), 170.8 (C, C-8), 144.9 (C, aromatic), 140.6 (C, 2C, aromatics), 139.5 (C, aromatic), 126.5 (CH, aromatic), $126.3(\mathrm{CH}$, aromatic), $126.0(\mathrm{CH}, 2 \mathrm{C}$, aromatics), $125.1(\mathrm{CH}$, aromatic), $123.2(\mathrm{CH}$, aromatic $), 122.5(\mathrm{CH}$, aromatic), $121.3\left(\mathrm{CH}\right.$, aromatic), $99.5(\mathrm{CH}, \mathrm{C}-1), 77.0(\mathrm{CH}, \mathrm{C}-5), 68.8\left(\mathrm{CH}_{2}, \mathrm{C}-6\right), 63.0$ $\left(\mathrm{CH}_{2}, \mathrm{C}-7\right), 50.1$ (CH, C-4a), 48.5 (C, C-3a), $44.3(\mathrm{CH}, \mathrm{C}-4), 44.0(\mathrm{CH}, \mathrm{C}-3), 20.9$ $\left(\mathrm{CH}_{3}, \mathrm{C}-9\right)$; HRMS calc. for $\mathrm{C}_{23} \mathrm{H}_{21} \mathrm{O}_{5}[\mathrm{M}+\mathrm{H}]^{+}$377.1389. Found 377.1374.

\section{Diels-Alder Product 5d}

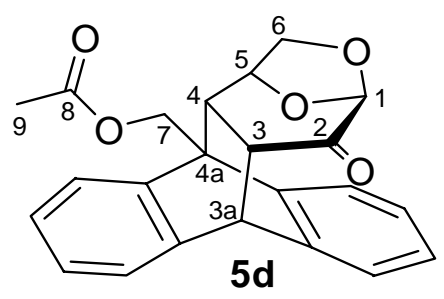

Colorless oil; $[\alpha]^{25}-79.3$ (c 1.45, $\mathrm{CHCl}_{3}$ ); IR (film) 3072, 2967, 2902, 1739 (C=O), $1458,1234,1117,1043,751 \mathrm{~cm}^{-1} ;{ }^{1} \mathrm{H} \mathrm{NMR}\left(\mathrm{CDCl}_{3}\right) \delta 7.40-7.09(\mathrm{~m}, 8 \mathrm{H}$, aromatics), $5.41(\mathrm{~s}, 2 \mathrm{H}, \mathrm{H}-7), 4.87\left(\mathrm{~d}, J_{3-3 \mathrm{a}}=3.3 \mathrm{~Hz}, 1 \mathrm{H}, \mathrm{H}-3 \mathrm{a}\right), 4.84\left(\mathrm{~d}, J_{5-6 \mathrm{exo}}=5.3 \mathrm{~Hz}, 1 \mathrm{H}, \mathrm{H}-5\right)$, $4.65(\mathrm{~s}, 1 \mathrm{H}, \mathrm{H}-1), 3.83$ (dd, $\left.J_{\text {gem }}=7.3 \mathrm{~Hz}, J_{5-6 \mathrm{exo}}=5.3 \mathrm{~Hz}, 1 \mathrm{H}, \mathrm{H}-6 \mathrm{exo}\right), 3.68$ (dd, $\left.J_{\text {gem }}=7.3 \mathrm{~Hz}, J_{5-6 \text { endo }}=1.0 \mathrm{~Hz}, 1 \mathrm{H}, \mathrm{H}-6 \mathrm{endo}\right), 3.02\left(\mathrm{dd}, J_{3-4}=10.0 \mathrm{~Hz}, J_{3-3 \mathrm{a}}=3.3 \mathrm{~Hz}, 1 \mathrm{H}\right.$, $\mathrm{H}-3), 2.39$ (d, $\left.J_{3-4}=10.0 \mathrm{~Hz}, 1 \mathrm{H}, \mathrm{H}-4\right), 2.19$ (s, $\left.3 \mathrm{H}, \mathrm{H}-9\right) ;{ }^{13} \mathrm{C} \mathrm{NMR}\left(\mathrm{CDCl}_{3}\right) \delta 198.4$ (C, C-2), 170.8 (C, C-8), 143.2 (C, aromatic), 141.6 (C, aromatic), 140.8 (C, aromatic), $139.4(\mathrm{C}$, aromatic), $126.4(\mathrm{CH}$, aromatic), $126.3(\mathrm{CH}$, aromatic), $126.2(\mathrm{CH}$, aromatic), $126.1(\mathrm{CH}$, aromatic), $125.0(\mathrm{CH}$, aromatic), $124.1(\mathrm{CH}$, aromatic $), 121.5(\mathrm{CH}, 2 \mathrm{C}$, aromatic), $99.3(\mathrm{CH}, \mathrm{C}-1), 72.3(\mathrm{CH}, \mathrm{C}-5), 69.3\left(\mathrm{CH}_{2}, \mathrm{C}-6\right), 62.1\left(\mathrm{CH}_{2}, \mathrm{C}-7\right), 47.1(\mathrm{C}$, C-4a), 46.4 (CH, C-3a), 45.7 (CH, C-3), 43.8 (CH, C-4), $20.8\left(\mathrm{CH}_{3}, \mathrm{C}-9\right)$; HRMS calc. for $\mathrm{C}_{23} \mathrm{H}_{20} \mathrm{O}_{5}[\mathrm{M}]^{+}$376.1311. Found 376.1292. 


\section{Diels-Alder Product 4e}

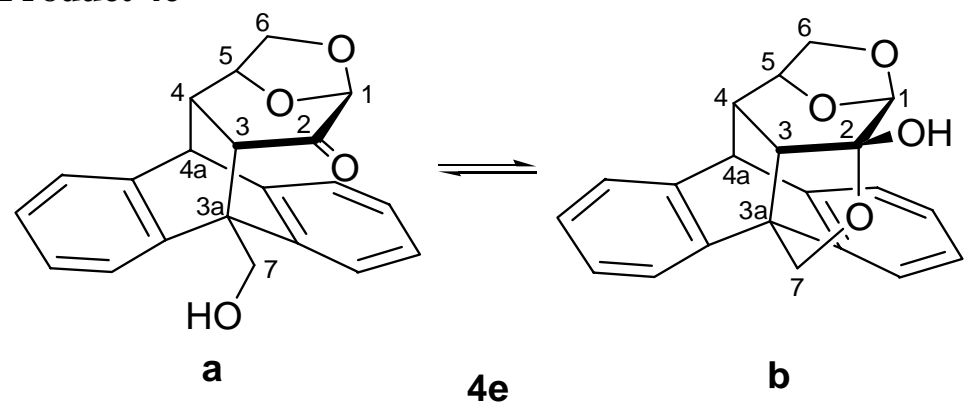

Colorless oil; IR (film) 3442, 3040, 2958, 1725 (C=O), 1457, 1245, 1118, 1083, 1045, 993, $749 \mathrm{~cm}^{-1} ;{ }^{1} \mathrm{H}$ NMR $\left(\mathrm{CDCl}_{3}\right) \delta 7.67-6.99(\mathrm{~m}$, aromatics of $\mathbf{a}$ and $\mathbf{b}), 5.06(\mathrm{~d}$, $J_{\text {gem }}=9.2 \mathrm{~Hz}, \mathrm{H}-7$ of b), $4.90\left(\mathrm{~d}, J_{\text {gem }}=12.3 \mathrm{~Hz}, \mathrm{H}-7\right.$ of a), $4.82\left(\mathrm{~d}, J_{\text {gem }}=12.3 \mathrm{~Hz}, \mathrm{H}-7\right.$ of a), $4.74(\mathrm{~d}, J=9.2 \mathrm{~Hz}, \mathrm{H}-7$ of b), 4.74 (broad s, H-5 of a and H-1 of b), 4.62 (s, H-1 of a), $4.38\left(\mathrm{~d}, J_{4-4 \mathrm{a}}=4.1 \mathrm{~Hz}, \mathrm{H}-4 \mathrm{a}\right.$ of $\left.\mathbf{b}\right), 4.33\left(\mathrm{~d}, J_{4-4 \mathrm{a}}=1.9 \mathrm{~Hz}, \mathrm{H}-4 \mathrm{a}\right.$ of a), $4.21\left(\mathrm{~d}, J_{5-}\right.$ ${ }_{6 e x o}=5.4 \mathrm{~Hz}, \mathrm{H}-5$ of b), 3.77-3.54 (m, H-6 of a and b), $3.08\left(\mathrm{~d}, J_{3-4}=9.9 \mathrm{~Hz}, \mathrm{H}-3\right.$ of a), $2.47\left(\mathrm{~d}, J_{3-4}=10.3 \mathrm{~Hz}, \mathrm{H}-3\right.$ of $\left.\mathbf{b}\right), 2.34-2.24(\mathrm{~m}, \mathrm{H}-4$ of $\mathbf{a}$ and $\mathbf{b}) ;{ }^{13} \mathrm{C} \mathrm{NMR}\left(\mathrm{CDCl}_{3}\right) \delta$ 201.3 (C, C-2 of $\mathbf{a}), 144.6$ (C, aromatic of $\mathbf{a}$ and $\mathbf{b}), 143.8$ (C, aromatic of $\mathbf{a}$ and $\mathbf{b}$ ), $142.2(\mathrm{C}$, aromatic of $\mathbf{a}$ and $\mathbf{b}), 141.4(\mathrm{C}$, aromatic of $\mathbf{a}$ and $\mathbf{b}), 140.8(\mathrm{C}$, aromatic of $\mathbf{a}$ and $\mathbf{b}), 139.9(\mathrm{C}$, aromatic of $\mathbf{a}$ and $\mathbf{b}), 126.5(\mathrm{CH}$, aromatic of $\mathbf{a}$ and $\mathbf{b}), 126.0(\mathrm{CH}$, aromatic of $\mathbf{a}$ and $\mathbf{b}), 125.6(\mathrm{CH}$, aromatic of $\mathbf{a}$ and $\mathbf{b}), 125.4(\mathrm{CH}$, aromatic of $\mathbf{a}$ and b), $124.8(\mathrm{CH}$, aromatic of $\mathbf{a}$ and $\mathbf{b}), 124.5(\mathrm{CH}$, aromatic of $\mathbf{a}$ and $\mathbf{b}), 123.7(\mathrm{CH}$, aromatic of $\mathbf{a}$ and $\mathbf{b}), 123.3(\mathrm{CH}$, aromatic of $\mathbf{a}$ and $\mathbf{b}), 122.8(\mathrm{CH}$, aromatic of $\mathbf{a}$ and b), $122.2(\mathrm{CH}$, aromatic of $\mathbf{a}$ and $\mathbf{b}), 120.9(\mathrm{CH}$, aromatic of $\mathbf{a}$ and $\mathbf{b}), 118.5(\mathrm{CH}$, aromatic of $\mathbf{a}$ and $\mathbf{b}), 99.8(\mathrm{CH}, \mathrm{C}-1$ of $\mathbf{a}), 98.8(\mathrm{CH}, \mathrm{C}-1$ of $\mathbf{b}), 98.8(\mathrm{C}, \mathrm{C}-2 \mathrm{of} \mathbf{b}), 76.8$ $(\mathrm{CH}, \mathrm{C}-5$ of $\mathbf{a}), 73.5(\mathrm{CH}, \mathrm{C}-5$ of $\mathbf{b}), 69.7\left(\mathrm{CH}_{2}, \mathrm{C}-6\right.$ of $\left.\mathbf{b}\right), 69.0\left(\mathrm{CH}_{2}, \mathrm{C}-6\right.$ of $\left.\mathbf{a}\right), 65.7$ $\left(\mathrm{CH}_{2}, \mathrm{C}-7\right.$ of b), $60.6\left(\mathrm{CH}_{2}, \mathrm{C}-7\right.$ of a), $56.3(\mathrm{CH}, \mathrm{C}-4 \mathrm{a}$ of $\mathbf{b}), 56.2(\mathrm{C}, \mathrm{C}-3 \mathrm{a}$ of $\mathbf{b}), 50.9$ $(\mathrm{C}, \mathrm{C}-3 \mathrm{a}$ of $\mathbf{a}), 50.2(\mathrm{CH}, \mathrm{C}-4 \mathrm{a}$ of $\mathbf{a}), 50.0(\mathrm{CH}, \mathrm{C}-4$ of $\mathbf{b}), 44.6(\mathrm{CH}, \mathrm{C}-4 \mathrm{do} \mathbf{a}), 42.5$ $\left(\mathrm{CH}, \mathrm{C}-3\right.$ of a), $37.7\left(\mathrm{CH}, \mathrm{C}-3\right.$ of b); HRMS calc. for $\mathrm{C}_{21} \mathrm{H}_{19} \mathrm{O}_{4}[\mathrm{M}+\mathrm{H}]^{+} 335.1283$. Found 335.1276.

\section{Diels-Alder Product 5e}

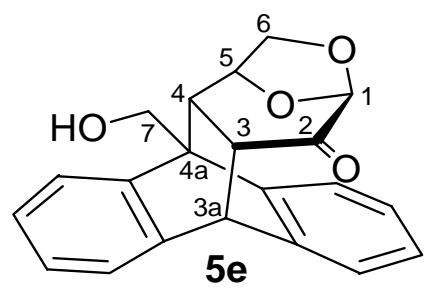

Colorless oil; $[\alpha]^{25}$-76.7 (c 1.02, $\mathrm{CHCl}_{3}$ ); IR (film) 3511, 3023, 2967, 2900, 1724 $(\mathrm{C}=\mathrm{O}), 1458,1118,985,751 \mathrm{~cm}^{-1} ;{ }^{1} \mathrm{H}$ NMR $\left(\mathrm{CDCl}_{3}\right) \delta 7.50-7.05(\mathrm{~m}, 8 \mathrm{H}$, aromatics $)$, $4.99\left(\mathrm{~d}, J_{\mathrm{gem}}=11.1 \mathrm{~Hz}, 1 \mathrm{H}, \mathrm{H}-7\right), 4.96\left(\mathrm{~d}, J_{5-6 \mathrm{exo}}=5.5 \mathrm{~Hz}, 1 \mathrm{H}, \mathrm{H}-5\right), 4.91\left(\mathrm{~d}, J_{\text {gem }}=11.1\right.$ $\mathrm{Hz}, 1 \mathrm{H}, \mathrm{H}-7), 4.85$ (d, $\left.J_{3-3 \mathrm{a}}=3,3 \mathrm{~Hz}, 1 \mathrm{H}, \mathrm{H}-3 \mathrm{a}\right), 4.64$ (s, $\left.1 \mathrm{H}, \mathrm{H}-1\right), 3.80$ (dd, $J_{5-6 \text { exo }}=5.5$ $\left.\mathrm{Hz}, J_{\text {gem }}=7.4 \mathrm{~Hz}, 1 \mathrm{H}, \mathrm{H}-6 \mathrm{exo}\right), 3.67\left(\mathrm{dd}, J_{\text {gem }}=7.4 \mathrm{~Hz}, J_{5-6 e n d o}=1.1 \mathrm{~Hz}, 1 \mathrm{H}, \mathrm{H}-6 e n d o\right)$, $2.99\left(\mathrm{dd}, J_{3-4}=10.0 \mathrm{~Hz}, J_{3-3 \mathrm{a}}=3.3 \mathrm{~Hz}, 1 \mathrm{H}, \mathrm{H}-3\right), 2.43\left(\mathrm{~d}, J_{3-4}=10.0 \mathrm{~Hz}, 1 \mathrm{H}, \mathrm{H}-4\right), 2.21$ (broad s, $1 \mathrm{H}, \mathrm{OH}) ;{ }^{13} \mathrm{C} \mathrm{NMR}\left(\mathrm{CDCl}_{3}\right) \delta 199.2(\mathrm{C}, \mathrm{C}-2), 144.0$ (C, aromatic), $141.7(\mathrm{C}$, aromatic), $141.2(\mathrm{C}$, aromatic), $140.3(\mathrm{C}$, aromatic), $126.2(\mathrm{CH}$, aromatic), $126.0(\mathrm{CH}$, $2 \mathrm{C}$, aromatics), $125.9(\mathrm{CH}$, aromatic), $124.8(\mathrm{CH}$, aromatic), $124.2(\mathrm{CH}$, aromatic), $121.5(\mathrm{CH}$, aromatic), $121.4(\mathrm{CH}$, aromatic), $99.3(\mathrm{CH}, \mathrm{C}-1), 72.5(\mathrm{CH}, \mathrm{C}-5), 69.3$ 
$\left(\mathrm{CH}_{2}, \mathrm{C}-6\right), 60.1\left(\mathrm{CH}_{2}, \mathrm{C}-7\right), 48.3(\mathrm{C}, \mathrm{C}-4 \mathrm{a}), 46.3(\mathrm{CH}, \mathrm{C}-3 \mathrm{a}), 45.4(\mathrm{CH}, \mathrm{C}-3), 43.2$ $(\mathrm{CH}, \mathrm{C}-4)$.

\section{Acetylation of $4 \mathrm{e}$}

The ortho adduct $4 \mathbf{e}(105 \mathrm{mg}, 0.314 \mathrm{mmol}$ ) (as a mixture of the free carbonyl compound and the corresponding hemiketal derivative) was dissolved in dry $\mathrm{CH}_{2} \mathrm{Cl}_{2}(2.5 \mathrm{~mL})$ under argon. The solution was cooled at $0{ }^{\circ} \mathrm{C}$ and dry pyridine $(0.36 \mathrm{~mL}, 4.45 \mathrm{mmol})$, DMAP $(3.9 \mathrm{mg}, 0.03 \mathrm{mmol})$ and $\mathrm{Ac}_{2} \mathrm{O}(0.28 \mathrm{~mL}, 2.94 \mathrm{mmol})$ were added in this order. The solution was stirred for 1 hour at $0{ }^{\circ} \mathrm{C}$. The solvent was evaporated under reduced pressure and the crude was dissolved with AcOEt $(100 \mathrm{~mL})$. The organic phase was washed with saturated $\mathrm{CuSO}_{4}(3 \times 100 \mathrm{~mL})$ and brine $(2 \times 100 \mathrm{~mL})$, dried $\left(\mathrm{Na}_{2} \mathrm{SO}_{4}\right)$ and concentrated. The residual solid was purified by flash chromatography to afford $\mathbf{4 d}$ (59.1 mg, $0.157 \mathrm{mmol}, 50 \%)$ and $6 \mathbf{e}(32.9 \mathrm{mg}, 0.087 \mathrm{mmol}, 28 \%)$.

\section{Acetylated Diels-Alder Product 6e}

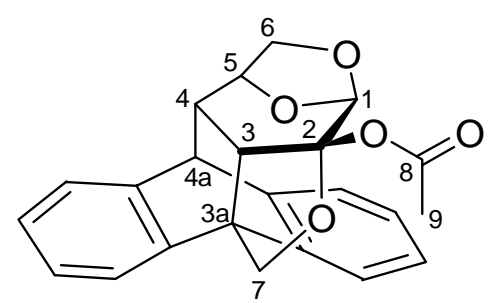

$6 e$

White solid; mp 238-239 ${ }^{\circ} \mathrm{C}$ (hexane/chloroform); $[\alpha]^{16}{ }_{\mathrm{D}}-35.6\left(c 0.56, \mathrm{CHCl}_{3}\right)$; IR (KBr) 3015, 2959, $1744(\mathrm{C}=\mathrm{O}), 1450,1364,1244,1234,1215,1001,959,758 \mathrm{~cm}^{-1} ;{ }^{1} \mathrm{H}$ NMR $\left(\mathrm{CDCl}_{3}\right) \delta$ 7.39-7.03 (m, $8 \mathrm{H}$, aromatics), $5.44(\mathrm{~s}, 1 \mathrm{H}, \mathrm{H}-1), 5.16\left(\mathrm{~d}, J_{\text {gem }}=9.1 \mathrm{~Hz}\right.$, $1 \mathrm{H}, \mathrm{H}-7), 4.68$ (d, $\left.J_{\text {gem }}=9.1 \mathrm{~Hz}, 1 \mathrm{H}, \mathrm{H}-7\right), 4.40\left(\mathrm{~d}, J_{4-4 \mathrm{a}}=4.0 \mathrm{~Hz}, 1 \mathrm{H}, \mathrm{H}-4 \mathrm{a}\right), 4.22$ (dd, $\left.J_{5-6 \text { exo }}=5.8 \mathrm{~Hz}, J_{5 \text {-6endo }}=1.3 \mathrm{~Hz}, 1 \mathrm{H}, \mathrm{H}-5\right), 3.68\left(\mathrm{dd}, J_{\text {gem }}=7.3 \mathrm{~Hz}, J_{5-6 \text { exo }}=5.8 \mathrm{~Hz}, 1 \mathrm{H}, \mathrm{H}\right.$ 6 exo), $3.55\left(\mathrm{dd}, J_{\text {gem }}=7.3 \mathrm{~Hz}, J_{5-6 \mathrm{endo}}=1.3 \mathrm{~Hz}, 1 \mathrm{H}, \mathrm{H}-6 \mathrm{endo}\right), 2.60\left(\mathrm{~d}, J_{3-4}=10.3 \mathrm{~Hz}, 1 \mathrm{H}\right.$, H-3), 2.35 (dd, $\left.J_{3-4}=10.3 \mathrm{~Hz}, J_{4-4 \mathrm{a}}=4.0 \mathrm{~Hz}, 1 \mathrm{H}, \mathrm{H}-4\right), 2.01$ (s, $\left.3 \mathrm{H}, \mathrm{H}-9\right)$ ) ${ }^{13} \mathrm{C} \mathrm{NMR}$ $\left(\mathrm{CDCl}_{3}\right) \delta 169.7$ (C, C-8), 144.2 (C, aromatic), 141.4 (C, aromatic), 141.2 (C, aromatic), 140.1 (C, aromatic), $126.2(\mathrm{CH}$, aromatic), $126.0(\mathrm{CH}$, aromatic), $125.9(\mathrm{CH}$, aromatic), $125.7(\mathrm{CH}$, aromatic), $124.6(\mathrm{CH}$, aromatic), $123.8(\mathrm{CH}$, aromatic), $122.2(\mathrm{CH}$, aromatic), $118.6(\mathrm{CH}$, aromatic), $103.7(\mathrm{C}, \mathrm{C}-2), 98.9(\mathrm{CH}, \mathrm{C}-1), 73.5(\mathrm{CH}, \mathrm{C}-5), 69.7$ $\left(\mathrm{CH}_{2}, \mathrm{C}-6\right), 66.8\left(\mathrm{CH}_{2}, \mathrm{C}-7\right), 55.2(\mathrm{C}, \mathrm{C}-3 \mathrm{a}), 54.9(\mathrm{CH}, \mathrm{C}-4 \mathrm{a}), 49.9(\mathrm{CH}, \mathrm{C}-4), 37.9$ $(\mathrm{CH}, \mathrm{C}-3), 21.4\left(\mathrm{CH}_{3}, \mathrm{C}-9\right)$. 
${ }^{1} \mathrm{H}$ and ${ }^{13} \mathrm{C}$ NMR Spectra of $\mathbf{4 a}$
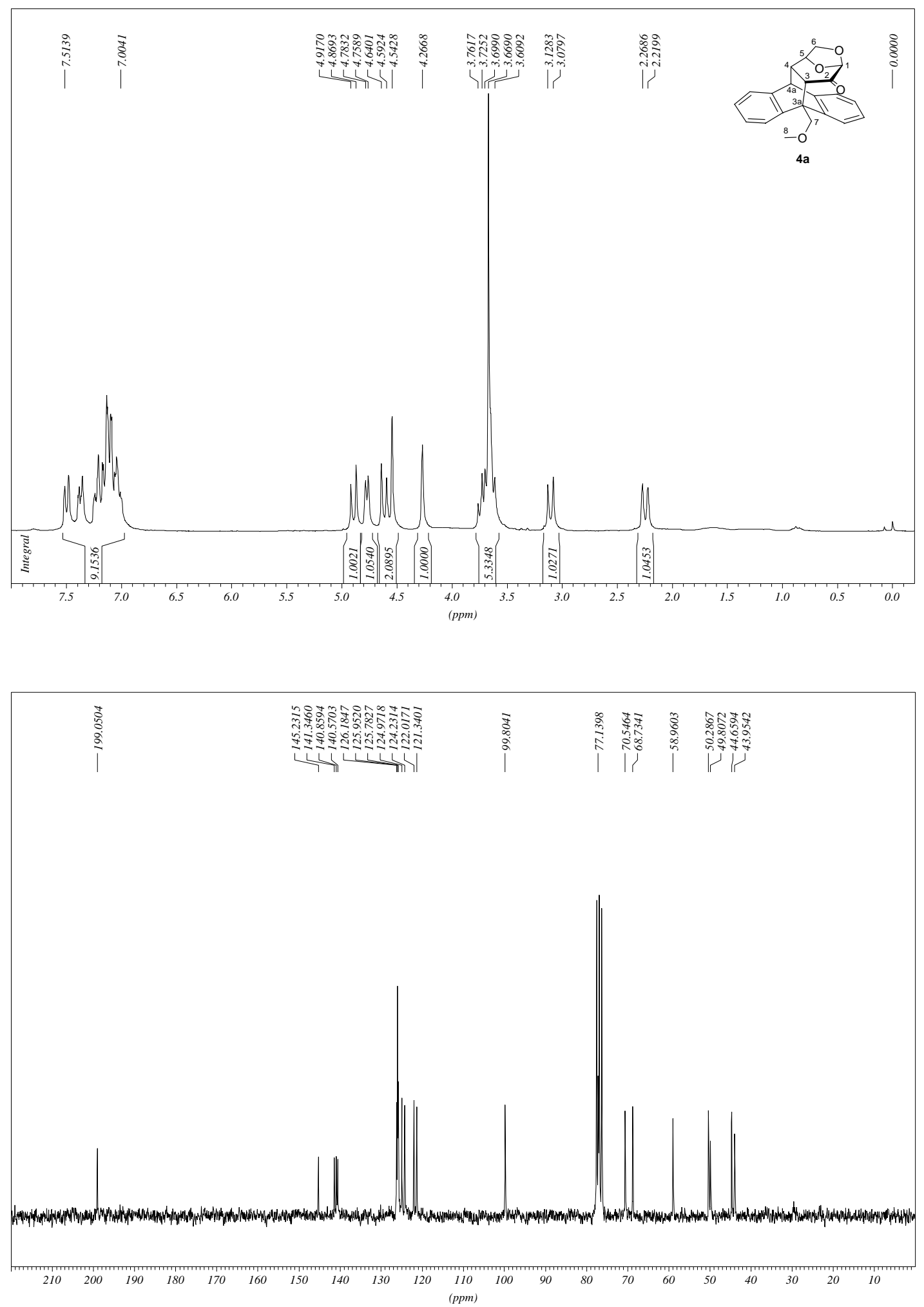
${ }^{1} \mathrm{H}$ and ${ }^{13} \mathrm{C}$ NMR Spectra of $\mathbf{5 a}$
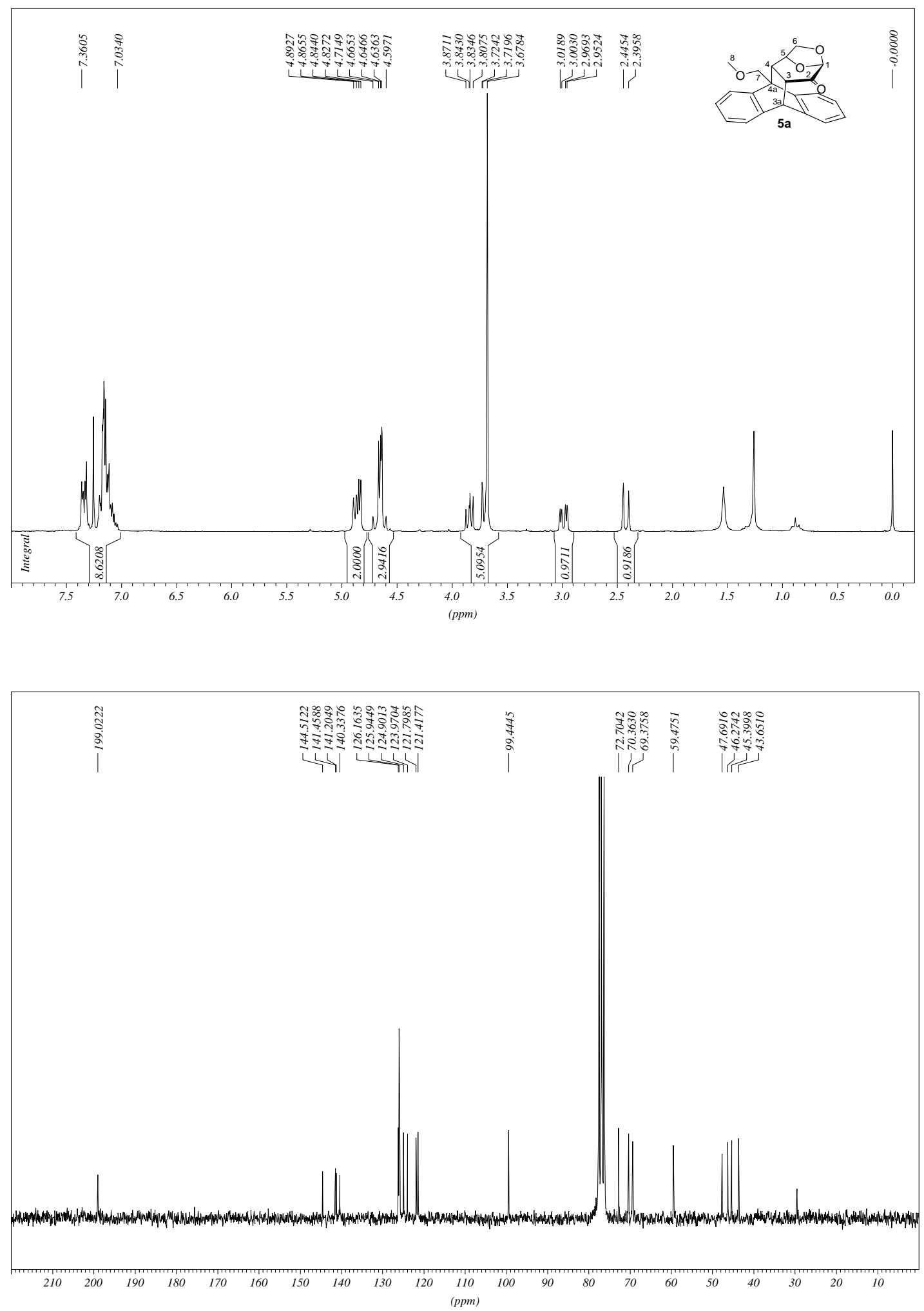
${ }^{1} \mathrm{H}$ and ${ }^{13} \mathrm{C}$ NMR Spectra of $\mathbf{4 b}$
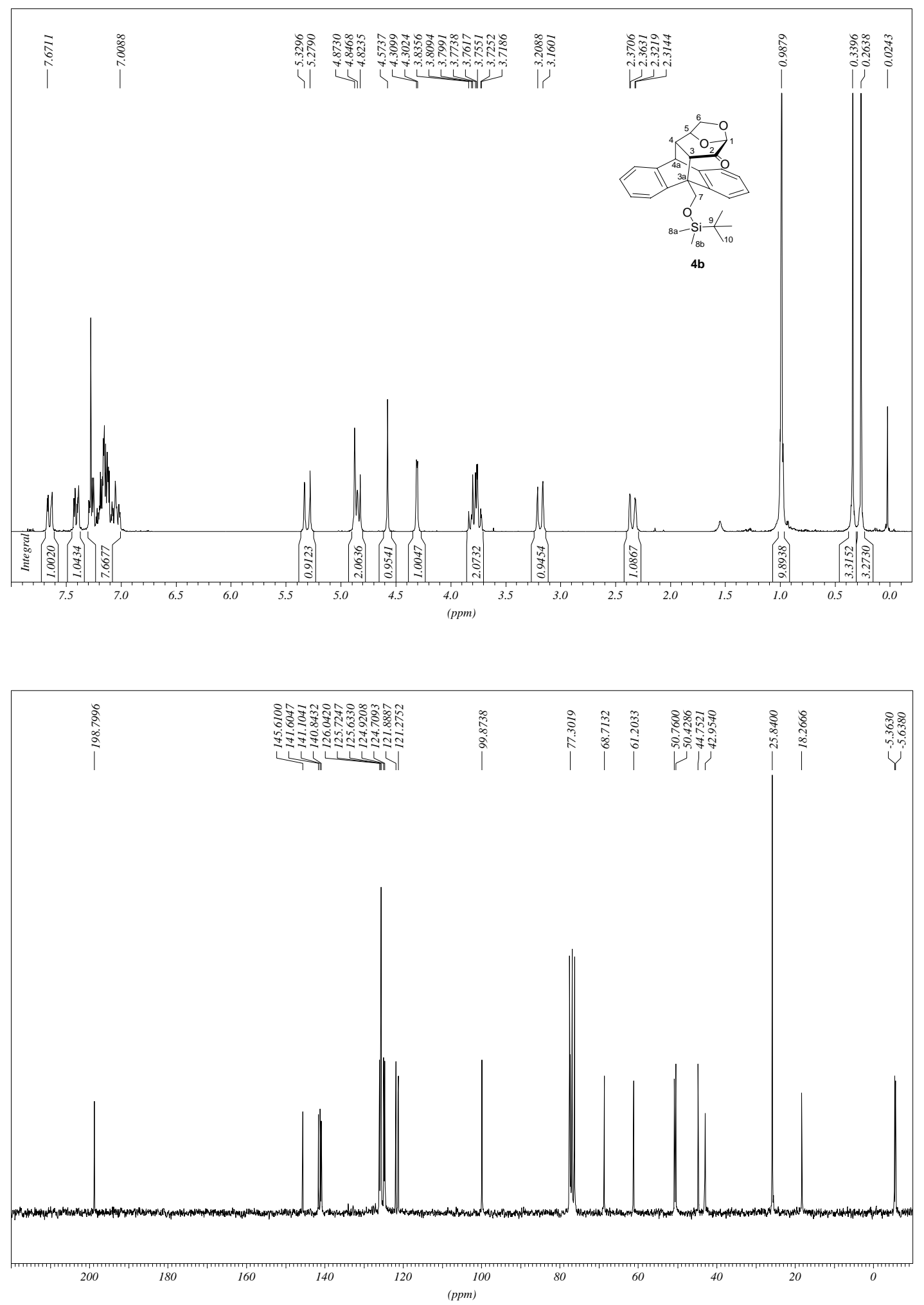
${ }^{1} \mathrm{H}$ and ${ }^{13} \mathrm{C}$ NMR Spectra of $\mathbf{5 b}$
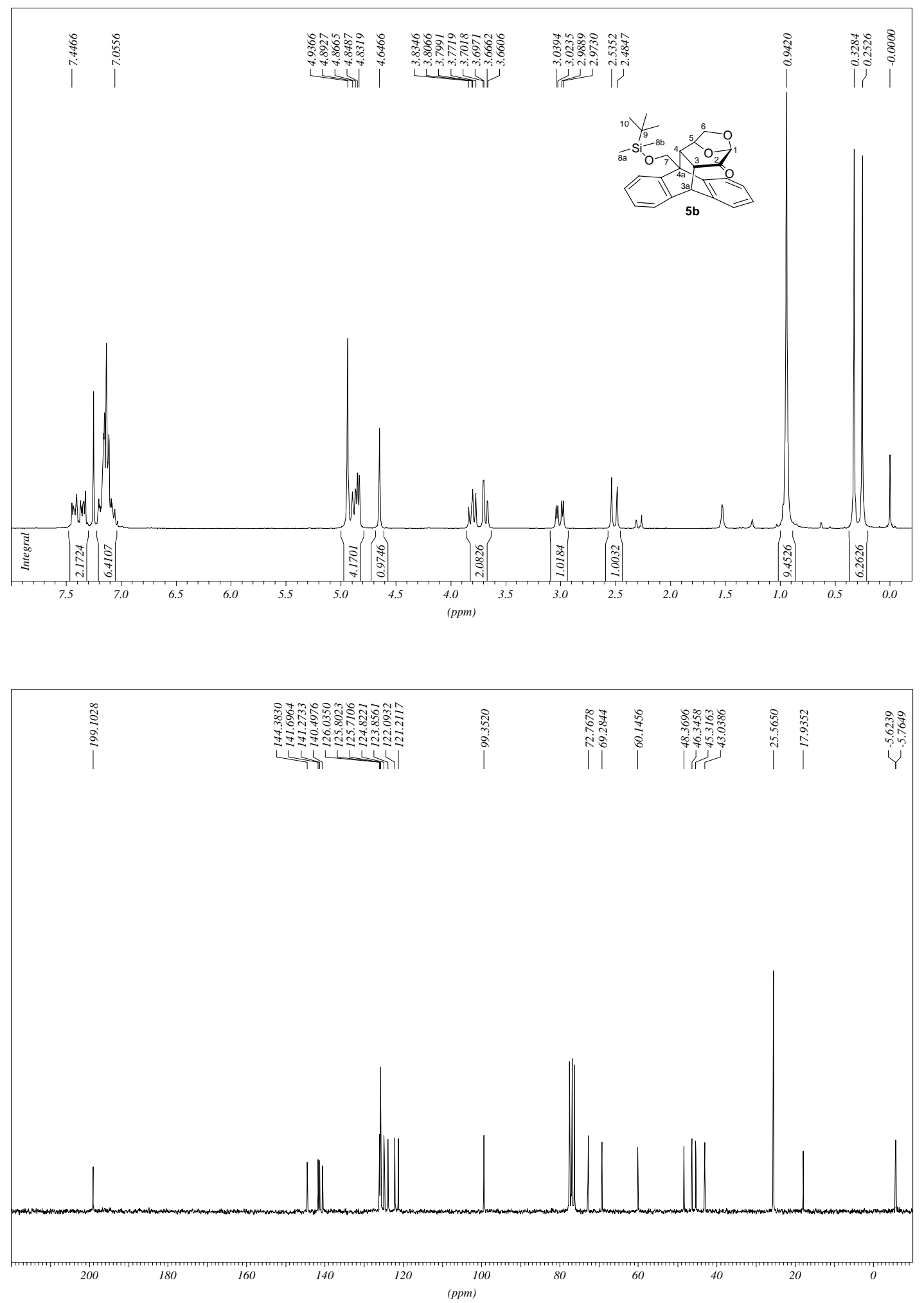
${ }^{1} \mathrm{H}$ and ${ }^{13} \mathrm{C}$ NMR Spectra of $\mathbf{4 c}$
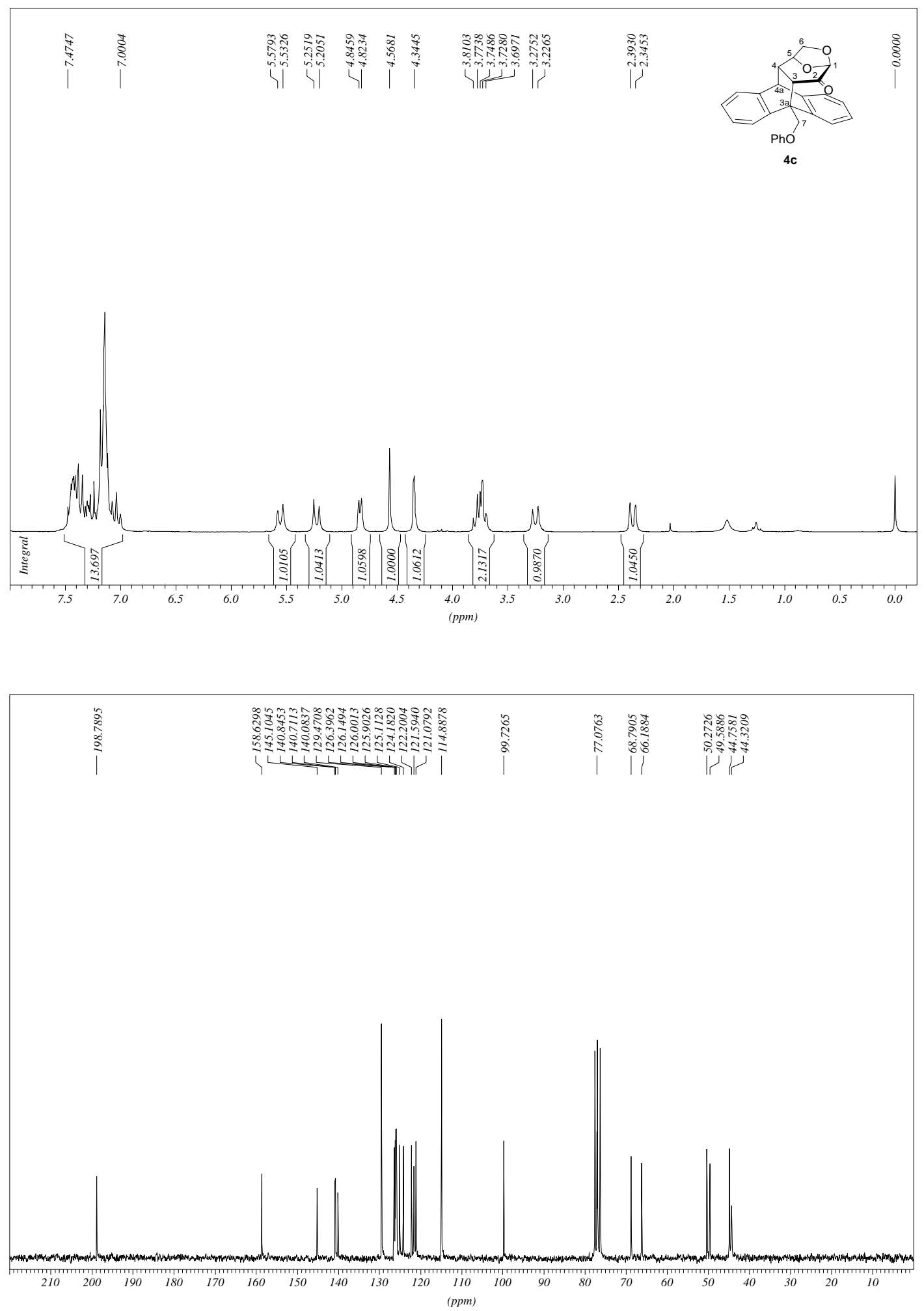
${ }^{1} \mathrm{H}$ and ${ }^{13} \mathrm{C}$ NMR Spectra of $\mathbf{4 d}$
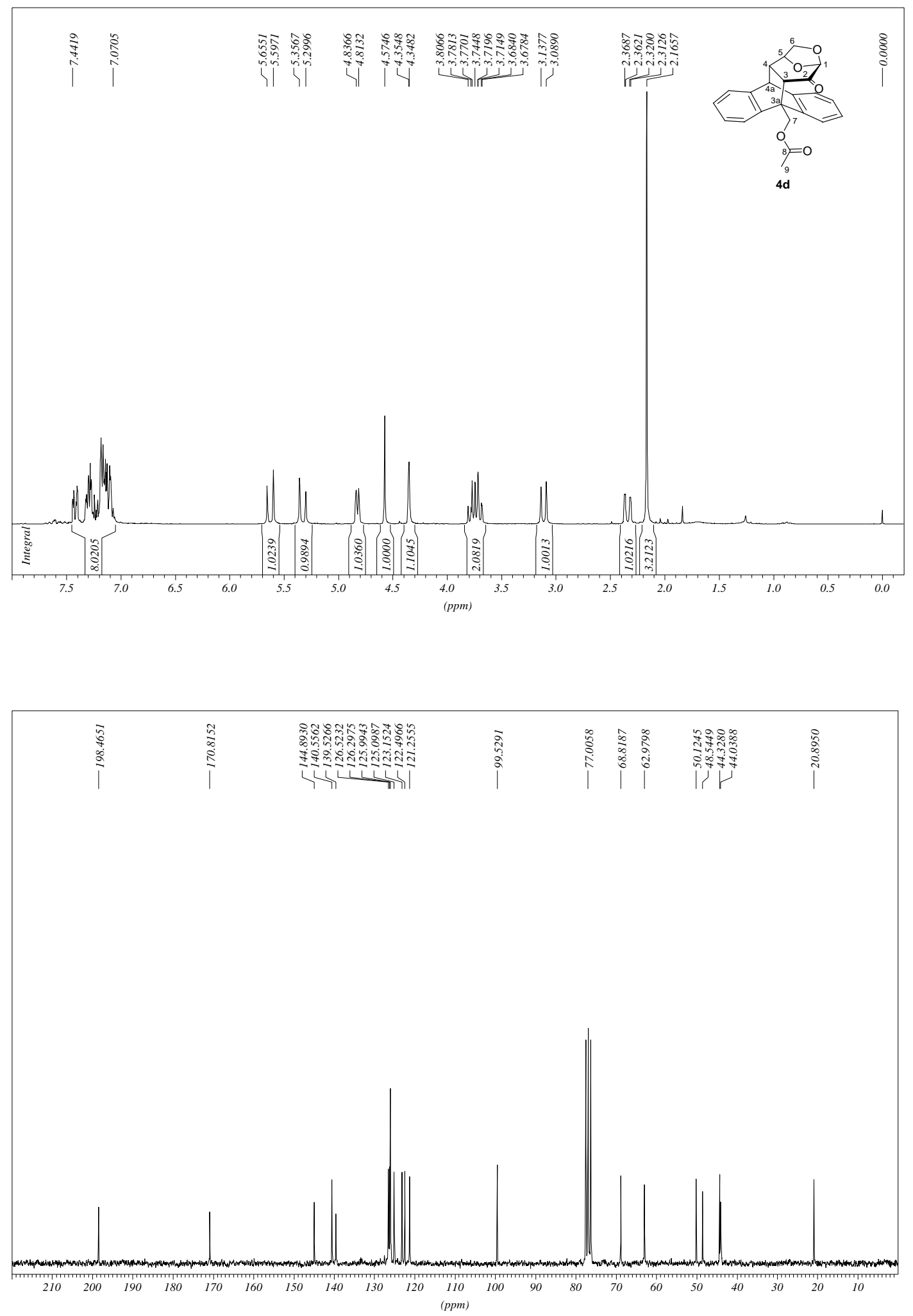
${ }^{1} \mathrm{H}$ and ${ }^{13} \mathrm{C}$ NMR Spectra of $\mathbf{5 d}$
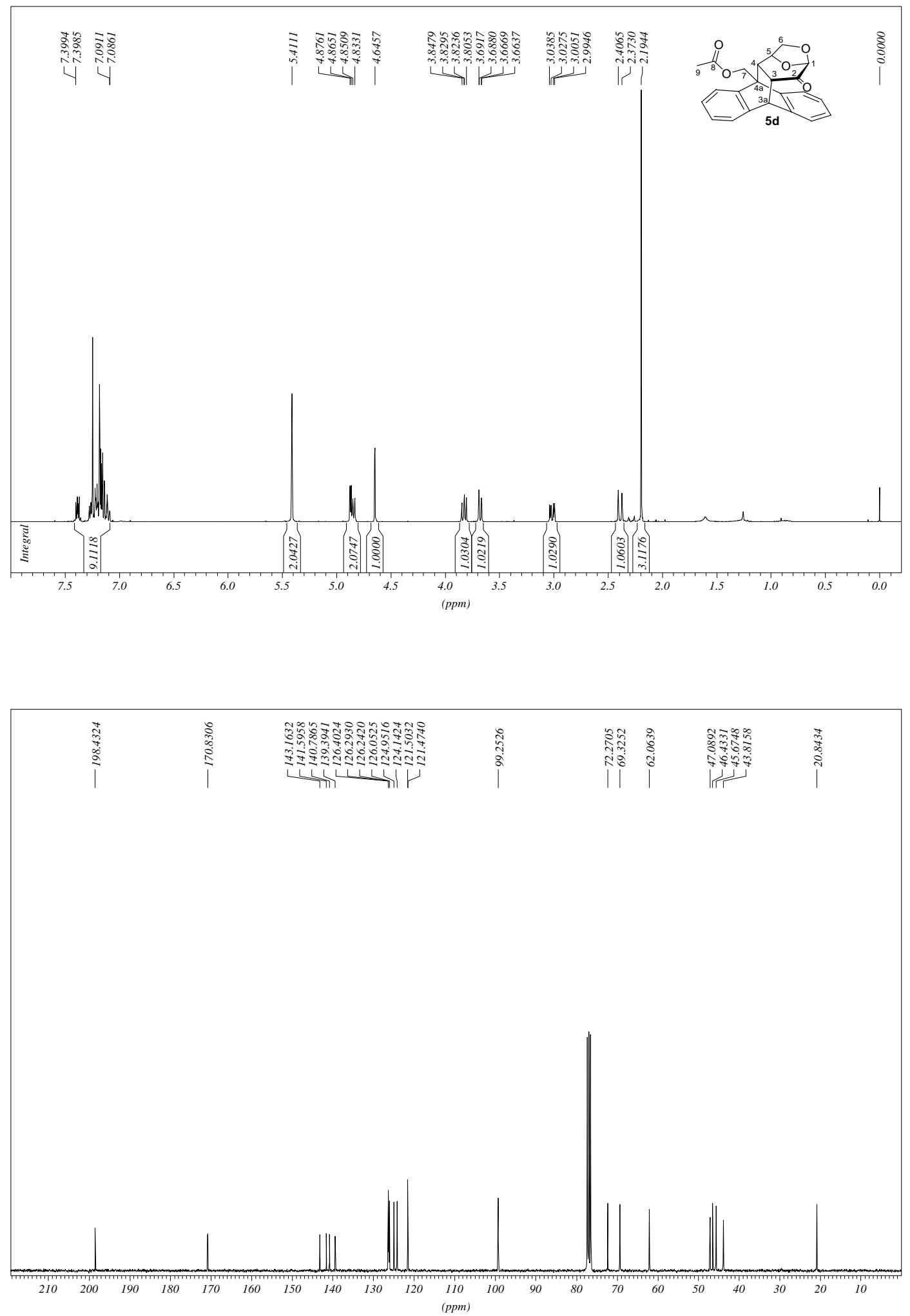
${ }^{1} \mathrm{H}$ and ${ }^{13} \mathrm{C}$ NMR Spectra of $\mathbf{4 e}$
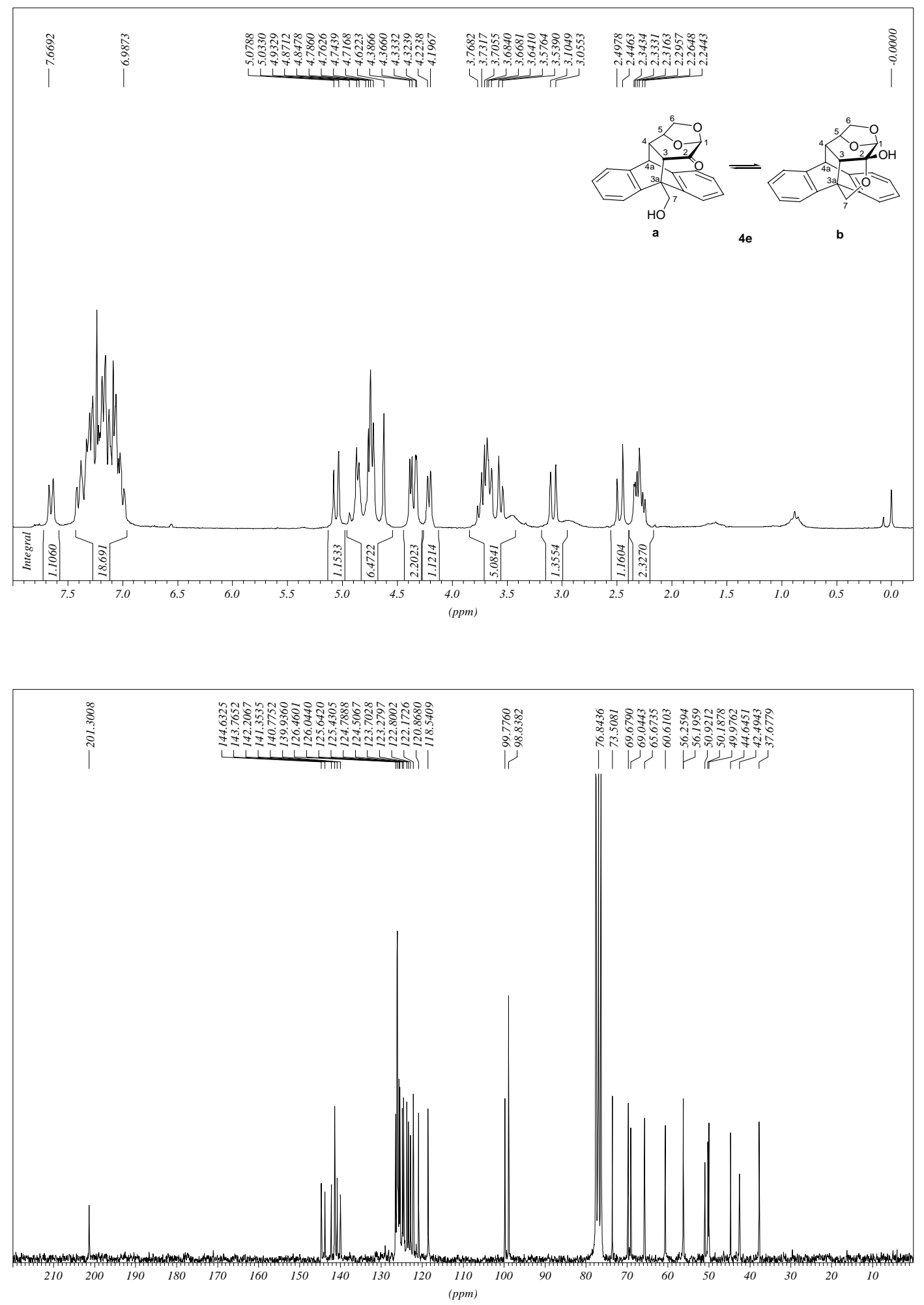
${ }^{1} \mathrm{H}$ and ${ }^{13} \mathrm{C}$ NMR Spectra of $\mathbf{5 e}$
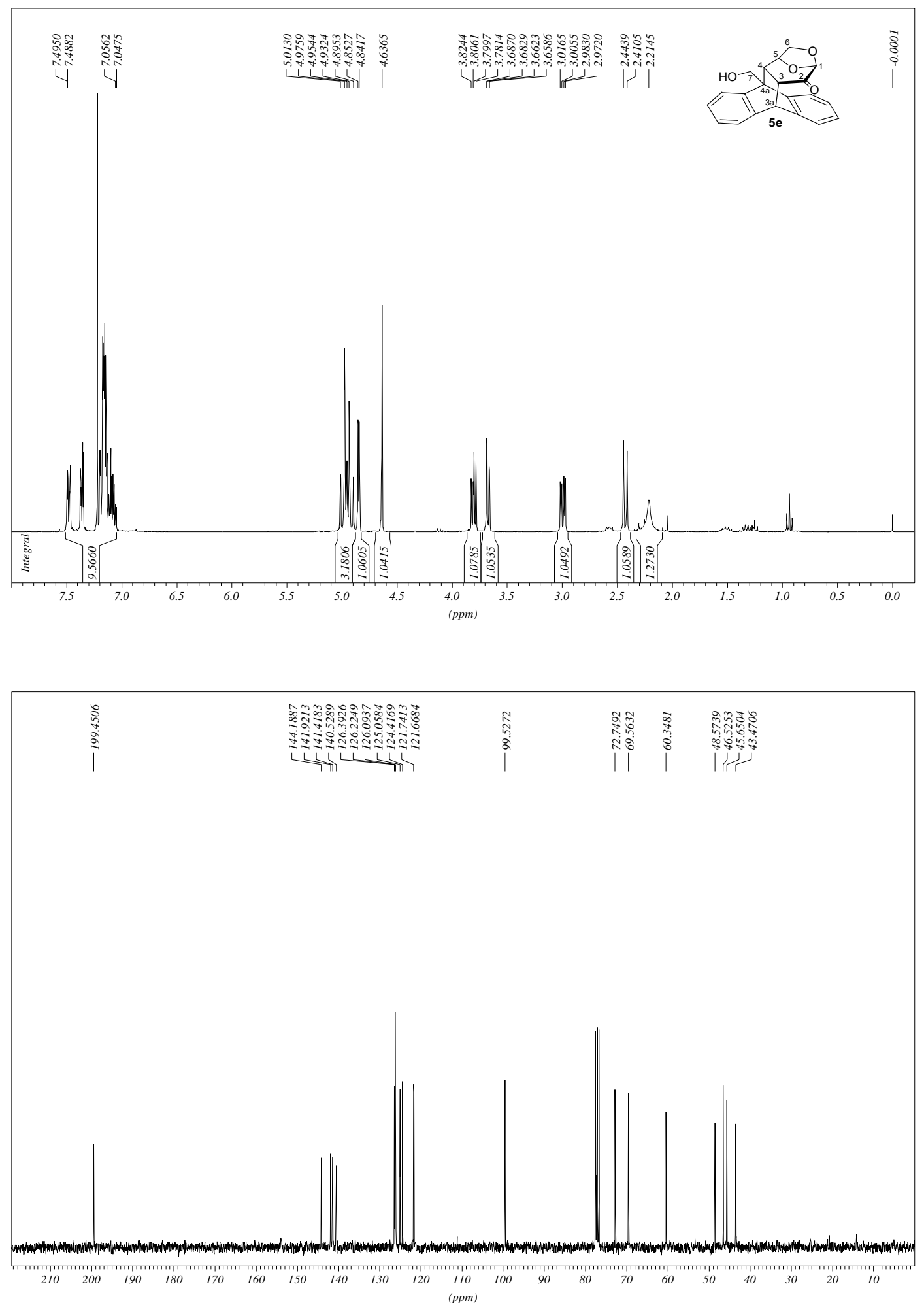
${ }^{1} \mathrm{H}$ and ${ }^{13} \mathrm{C}$ NMR Spectra of $6 \mathbf{e}$
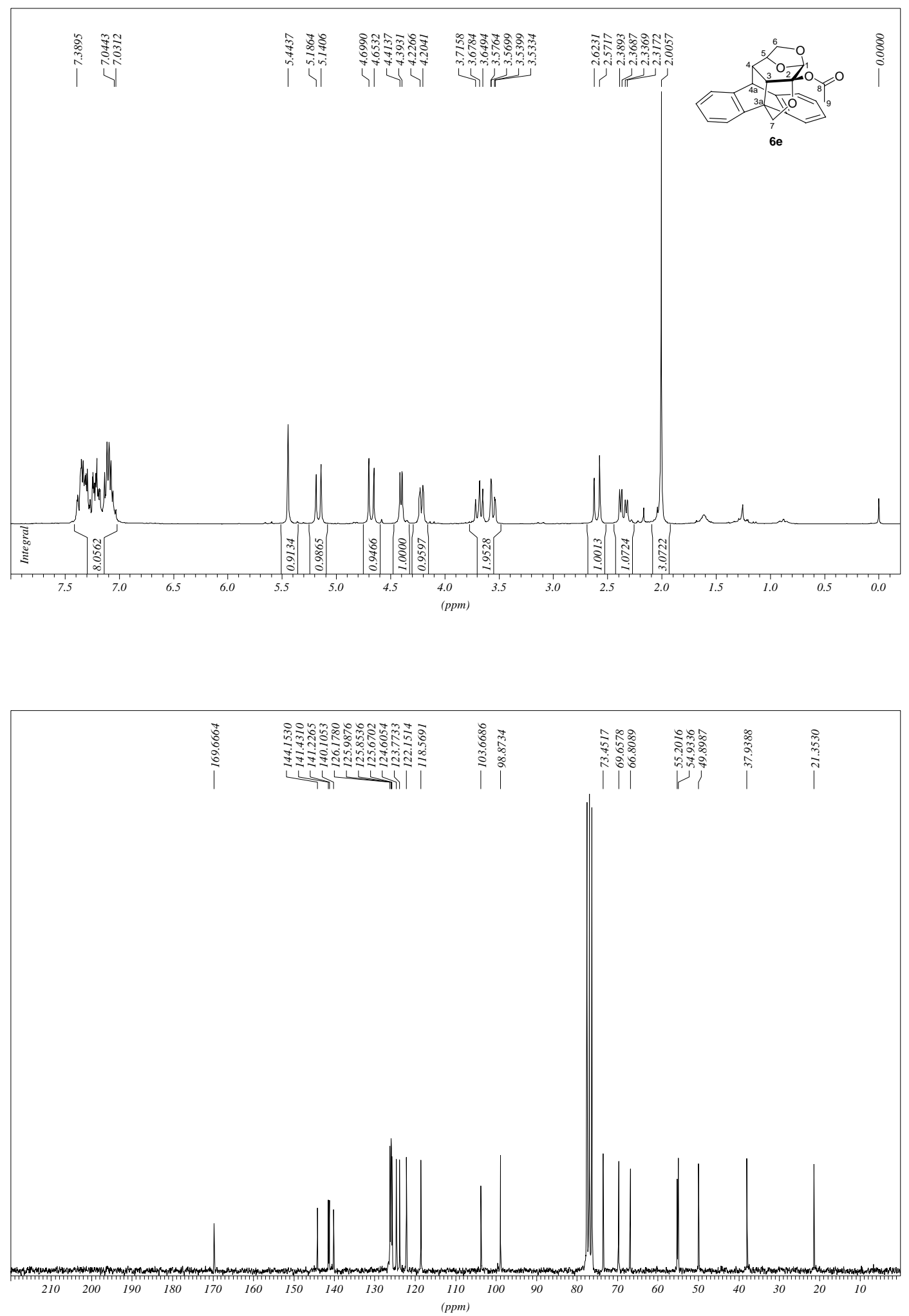